\title{
Article \\ Hydrogen Delocalization in an Asymmetric Biomolecule: The Curious Case of alpha-Fenchol
}

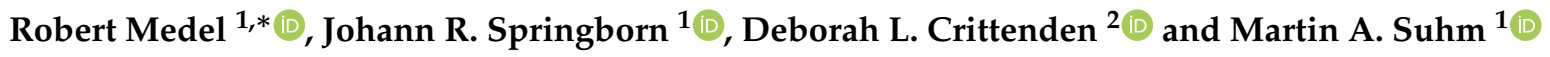 \\ 1 Institut für Physikalische Chemie, Georg-August-Universität Göttingen, Tammannstr. 6, 37077 Göttingen, \\ Germany \\ 2 School of Physical and Chemical Sciences, University of Canterbury, Private Bag 4800, Christchurch 8140, \\ New Zealand \\ * Correspondence: rmedel@gwdg.de
}

check for updates

Citation: Medel, R.; Springborn, J. R.; Crittenden D. L.; Suhm, M. A. Hydrogen Delocalization in an Asymmetric Biomolecule: The Curious Case of alpha-Fenchol. Preprints 2021, 1, 0. https://doi.org/

Received:

Accepted:

Published:

Publisher's Note: MDPI stays neutral with regard to jurisdictional claims in published maps and institutional affiliations.

\begin{abstract}
Rotational microwave jet spectroscopy studies of the monoterpenol $\alpha$-fenchol have so far failed to identify its second expected torsional conformer, despite computational predictions that it is only very slightly higher in energy than the most stable conformer. Vibrational FTIR and Raman jet spectroscopy investigations reveal unusually complex $\mathrm{OH}$ and OD stretching spectra compared to other alcohols. Via modelling of the torsional states, observed spectral splittings are explained by delocalization of the hydroxy hydrogen atom through quantum tunneling between the two non-equivalent but accidentally near-degenerate conformers separated by a low and narrow barrier. The energy differences between the torsional states are determined to be only 16(1) and $7(1) \mathrm{cm}^{-1} h c$ for the protiated and deuterated alcohol, respectively, which further shrink to 9(1) and $3(1) \mathrm{cm}^{-1} h c$ upon $\mathrm{OH}$ or OD stretch excitation. Comparisons are made with the more strongly asymmetric monoterpenols borneol and isopinocampheol as well as with the symmetric, rapidly tunneling propargyl alcohol. Assigned are also for $\alpha$-fenchol the third - in contrast localized torsional conformer and the most stable dimer, as well as for propargyl alcohol the two most stable dimers.
\end{abstract}

Keywords: delocalization; tunneling; terpene; alcohol; vibrational spectroscopy; supersonic jet expansion; structural determination

\section{Introduction}

In chemistry it is by now well recognized that quantum tunneling of particles between potential wells separated by finite barriers enhances reaction rates[1], but there is less awareness that it also can result in splitting of energy levels and delocalization[2]. The latter consequences are experimentally well characterized for coherent tunneling between equivalent wells in many small- to medium-sized systems in the gas phase or/and at cryogenic temperatures, e.g., in jet expansions. In contrast, biomolecular systems typically feature larger molecular structures or/and are affected by environmental perturbations, usually resulting in an absence of symmetry. However, localization with increasing asymmetry is a gradual, not abrupt, process. As a rule of thumb, the particle remains strongly delocalized if the introduced energetic asymmetry is not larger than the tunneling splitting in the symmetric case[3-5]. The description of tunneling in such slightly asymmetric double-minimum potentials continues to draw interest from the theoretical side.[6-14]

Known experimental splittings for hydrogen atom tunneling span a range of more than eight orders of magnitudes, with most of them being (far) smaller than $1 \mathrm{~cm}^{-1} \mathrm{hc}$ $\left(\approx 0.01 \mathrm{~kJ} \mathrm{~mol}^{-1}\right)[15]$. For this reason, localization is the typical, but not the strict, outcome from symmetry breaking by chemical[3,16-19] or isotopic $[4,5,20-28]$ substitution, internal rotation of molecular groups $[6,16,20,24,29]$, or from environmental influences such as solvation[16,30], matrix embedding [3,31,32] or crystallization[33]. Delocalization might be reintroduced if by excitation either the barrier[3,5,21,25,26,29] or the energetic asymmetry[22,34] is sufficiently lowered.

Only a few asymmetric systems with atoms substantially delocalized in the ground state are known. Among them are certain isotopologs of the well-studied dimer of hy- 
drogen chloride. The tunneling splittings from geared internal rotation of $\left(\mathrm{H}^{35} \mathrm{Cl}\right)_{2}$ and $\left(\mathrm{H}^{37} \mathrm{Cl}\right)_{2}$ are as large as 15.4 and $15.5 \mathrm{~cm}^{-1} h c$, respectively[35]. The zero-point asymmetry between hypothetical localized $\mathrm{H}^{35} \mathrm{Cl} \cdots \mathrm{H}^{37} \mathrm{Cl}$ and $\mathrm{H}^{37} \mathrm{Cl} \cdots \mathrm{H}^{35} \mathrm{Cl}$ isomers, differing in the acceptor $\cdots$ donor roles for the hydrogen bond, is calculated to be in the order of $1-2 \mathrm{~cm}^{-1} h c$. This is an order of magnitude smaller than the tunneling splitting, so that this isotopically mixed dimer remains highly delocalized. Still, the symmetry-breaking is sufficient to transfer spectral activity to additional transitions, which enabled the direct determination of the splittings in the ground and the $\mathrm{HCl}$ stretch excited states. In the latter the splitting is reduced to about $3.2 \mathrm{~cm}^{-1} h c$ for the symmetric dimers. Increase of the tunneling mass by double-deuteration reduces the tunneling splittings to 6.0 and $1.2 \mathrm{~cm}^{-1} \mathrm{hc}$ in the ground and $\mathrm{DCl}$ stretch excited states, respectively[36]. The latter is smaller than the asymmetry of $3.0 \mathrm{~cm}^{-1} \mathrm{hc}$ between the $\mathrm{DCl}$ stretch excited mixed $\mathrm{D}^{35} \mathrm{Cl} \cdots \mathrm{D}^{37} \mathrm{Cl}$ and $\mathrm{D}^{37} \mathrm{Cl} \cdots \mathrm{D}^{35} \mathrm{Cl}$ dimers, which are therefore better described as distinguishable localized species, in contrast to both the vibrational ground state and the protiated counterpart.

Symmetric parents for other well investigated structural families in respect to (partial) localization by chemical or isotopic symmetry breaking include porphycen (tunneling splitting of $\left.4.4 \mathrm{~cm}^{-1} \mathrm{hc}\right)$ [37-41], malonaldehyde $\left(21.6 \mathrm{~cm}^{-1} \mathrm{hc}\right)[4,7,14]$ and 9-hydroxyphenalenone $\left(69 \mathrm{~cm}^{-1} h c\right)[3,42-45]$.

Considering the vast structural diversity found in nature, it is perhaps not surprising, at least in hindsight, that tunneling splittings and partial delocalization also occur in inherently asymmetric biomolecules, i.e., systems which cannot be derived from a symmetric parent by minor modification. In this article we present spectroscopic and quantum-chemical evidence for this phenomenon in the monoterpenol $\alpha$-fenchol (Figure $1)$.

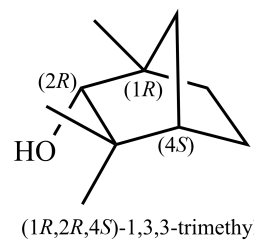

-bicyclo[2.2.1]heptan-2-ol

$(+)-\alpha$-fenchol

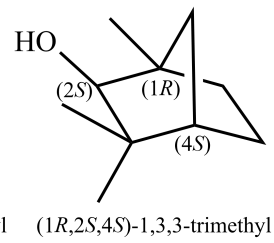

-bicyclo[2.2.1]heptan-2-ol

$(+)-\beta$-fenchol

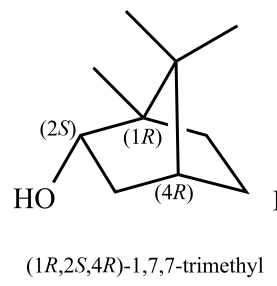

-bicyclo[2.2.1] heptan-2-ol

(+)-borneol

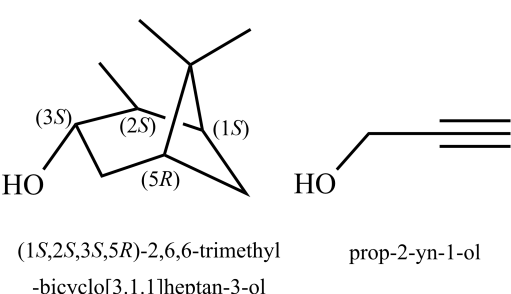

(+)-isopinocampheol propargyl alcohol

Figure 1. Structural formulas, systematic names and used trivial names of alcohols discussed in this study.

$\alpha$-Fenchol was previously investigated by Pate et al. in a neon expansion with microwave spectroscopy[46]. The three expected torsional conformers differ in the orientation of the light hydroxy hydrogen atom relative to the heavy and rigid molecular frame (Figure 2). A single dominant conformer was observed which was assigned to $g-$ based on the
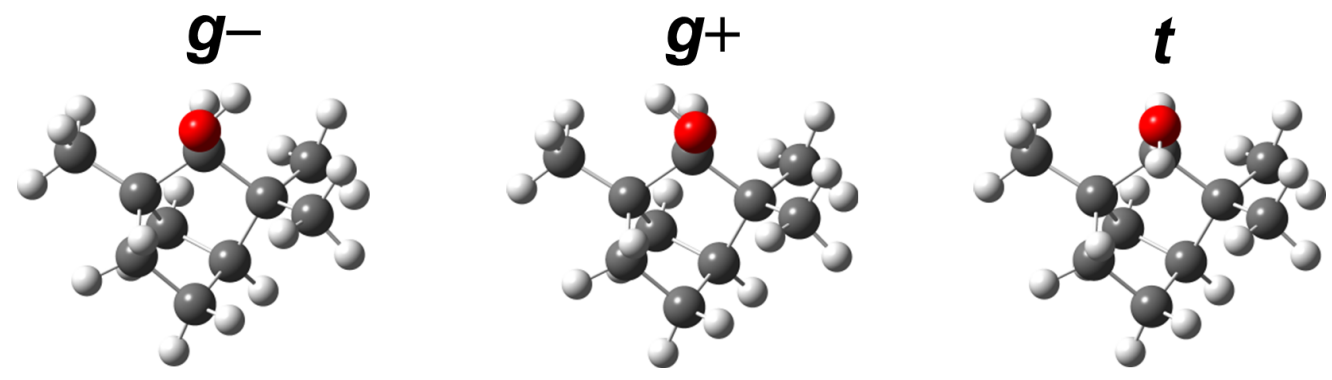

Figure 2. Optimized torsional conformers of (+)- $\alpha$-fenchol at B3LYP-D3(BJ)/may-cc-pVTZ level. They are labelled according to the qualitative arrangement of the $\mathrm{HOC}_{\alpha} \mathrm{H}$ dihedral: gauche $(g)$ $\approx-60^{\circ}$ or $+60^{\circ}$, or trans $(t) \approx \pm 180^{\circ}$.

good agreement between experimental and computed rotational constants, relative dipole moment components as well as its lowest predicted energy $\left(0.3 \mathrm{~kJ} \mathrm{~mol}^{-1}\right.$ below $g+$ at 
B3LYP-D3(BJ)/6-311++G(d,p) level). The calculated properties of the other conformers were not discussed, as the study focused on the determination of the enantiomeric excess via chiral tag spectroscopy.

A more definitive investigation of the diastereomerism of fenchol (including the epimer $\beta$-fenchol with the relative configuration at the alpha carbon atom inverted (Figure 1)), was done in parallel by Neeman and Huet, using the same experimental approach[47]. In contrast to Pate $e t$ al., they assessed that a conformational assignment for $\alpha$-fenchol on the basis of calculated rotational constants, dipole moment components and relative energy is not possible, as these are too similar between $g-$ and $g+$. Due to the challenges faced, they termed the conformational landscape a "maze" and nicely illustrated it as such in the table of content figure. Looking for further clues, they pointed out that the asymmetry parameter for the rotational constants agrees better with the prediction for the $g$ - conformer, a tendency which is further supported by the substitution geometry and the nuclear quadrupole hyperfine structure obtained after deuteration. So the same assignment as by Pate $e t$ al. was eventually concluded. Still, the absence of the second most stable $g+$ conformer, despite being predicted to be almost degenerate in energy (down to $0.03 \mathrm{~kJ} \mathrm{~mol}^{-1}$ at MP2 $/ 6-311++\mathrm{G}(\mathrm{d}, \mathrm{p})$ level), remained somewhat puzzling. It was even suspected that it does not represent a stable zero-point level in its torsional potential well due to the calculated low separating barrier.

Recently, we were able to assign all three expected conformers of both borneol and isopinocampheol[48], two constitutional isomers of $\alpha$-fenchol (Figure 1), by taking advantage of the differences in their $\mathrm{OH}$ stretching fundamental wavenumbers using Raman jet spectroscopy. In the present work, we extend our monoterpenol investigation to FTIR jet spectroscopy as well as $\alpha$-fenchol. The complexity of the obtained spectra for $\alpha$-fenchol is in stark contrast to the other two monoterpenols which indicates that the maze is even deeper than realized so far, but we also propose a non-classical way to escape it. As it turns out, there is not a single exit but one has to consider breaking through a barrier.

\section{Materials and Methods}

\subsection{Experimental techniques}

Gas mixtures were prepared by enriching 1.6 bar of helium with the vapor pressure of the respective compound at a set temperature. $\alpha$-Fenchol $\left(25^{\circ} \mathrm{C}, 96.8 \%\right.$, Alfa Aesar $),(-)$ borneol $\left(24{ }^{\circ} \mathrm{C}, 98.9 \%\right.$, Acros Organics), (-)-isopinocampheol $\left(24{ }^{\circ} \mathrm{C}, 98 \%\right.$, Sigma Aldrich) and propargyl alcohol $\left(-10\right.$ to $+23^{\circ} \mathrm{C}, 99 \%$, abcr $\left.\mathrm{GmbH}\right)$ were used as supplied. $\alpha-$ fenchol-OD was generated by dissolving the commercial sample in an excess of MeOD ( $99 \%$, Eurisotop), leaving the solution for half an hour for isotope exchange, evaporating methanol under reduced pressure and drying for 30 minutes in vacuum.

In the Raman jet setup[49] the respective gas mixture was expanded continuously at room temperature from a pressure between $0.7-0.8$ bar through a $(4 \times 0.15) \mathrm{mm}^{2}$ slit nozzle into an evacuated chamber. The expansion was probed at different distances from the nozzle by a Spectra Physics Millenia eV laser (532 nm, cw, 20-24 W). The scattered light was collected perpendicular to the propagation directions of both laser and jet with a camera lens and focused onto a one meter monochromator (McPherson). Photons from Stokes Raman scattering were co-added by a $\mathrm{LN}_{2}$-cooled CCD-camera (Princeton, PyLoN 400) over several minutes and averaged over multiple repetitions. The combination of laser and monochromator results in a spectral resolution of about $1 \mathrm{~cm}^{-1}$. The spectra were calibrated with neon vacuum transitions, we assume spectral positions and their differences to be accurate up to $\pm 1 \mathrm{~cm}^{-1}$.

In the FTIR jet setup[50] the gas mixture was expanded at a pressure between 0.10 1.20 bar through a $(600 \times 0.2) \mathrm{mm}^{2}$ slit nozzle into vacuum. The pulsed supersonic expansion was probed by a synchronized FTIR scan at $2 \mathrm{~cm}^{-1}$ resolution with a Bruker IFS $66 \mathrm{v} / \mathrm{S}$ spectrometer (single-sided-fast-return mode), with averaging over typically several hundred repetitions. For the recording of stationary vapor spectra at ambient temperature the same compound/helium gas mixtures (at a total pressure of $0.40 \mathrm{bar}$ ) and the same spectral 
parameters were used, averaged over one minute of background and one minute of sample scans. To correct for obstructing signals from drifting concentration of residual water vapor in the optical path (reduced by evacuation or flushing with dried air), a spectrum without sample was scaled to characteristic water lines in the $4000-3500 \mathrm{~cm}^{-1}$ region of each FTIR spectrum and subtracted.

\subsection{Computational techniques}

Density functional theory (DFT) and Møller-Plesset perturbation theory of second order (MP2) computations were carried out with the Gaussian 09 Rev. E.01 program package[51]. The B3LYP[52-55], PBE0[56] and B2PLYP[57] functionals together with Grimme's D3 dispersion correction without three-body term, with Becke-Johnson damping [58], the minimally augmented may-cc-pVTZ basis set[59], and the ultrafine integration grid were employed. MP2 was combined with the $6-311++G(d, p)$ basis set and tight convergence for the self consistent field. For geometry optimizations verytight convergence criteria were used with a subsequent frequency calculation in the double-harmonic approximation, in part with Raman activities. Minima were confirmed by the absence, and transition states by the presence of exactly one imaginary frequency. Single-point energies at the DLPNO-CCSD(T) level[60-62] with the aug-cc-pVQZ basis set were calculated on the DFT- or MP2-optimized geometries using ORCA version 4.2.1[63]. Relaxed scans of the electronic energy along a torsional dihedral angle $(\tau(\mathrm{HCOH})$ for methanol and secondary alcohols, $\tau(\mathrm{CCOH})$ for primary and tertiary alcohols) were performed at B3LYP-D3(BJ)/may-cc-pVTZ level with $1^{\circ}$ step size. For symmetric alcohols only the non-redundant part of the potential (e.g., $180^{\circ}$ for propargyl alcohol and $60^{\circ}$ for methanol) was scanned and then duplicated to preclude symmetry reduction by numerical noise. Used commands and keywords are available in the electronic supporting information (ESI).

For the simulation of spectra for localized conformers calculated Raman activities and depolarization ratios were converted to scattering cross sections. Accounted for are the laser wavelength and the polarization dependent sensitivity of monochromator and camera. This is detailed in the ESI. Calculated infrared band strengths were used directly. Relative populations are based on the assumption that the conformational cooling stops at about $100 \mathrm{~K}$ and that vibrational and rotational partition functions for the conformers are similar enough to justify the use of zero-point corrected relative energies instead of Gibbs energies. The value of $100 \mathrm{~K}$ corresponds to an estimate at a detection distance from the nozzle of $1 \mathrm{~mm}$ [64], using the accurately known conformational energy difference of ethanol[65].

Numerical solutions to the 1D torsional Schrödinger equation were obtained using the Chung-Phillips algorithm[66], employing reduced moments of inertia that account for minor counter-rotation of the alcohol scaffold upon hydroxy group rotation. The code is available on github, at https://github.com/dlc62/Torsion1D. To account for the dependence of the effective $\mathrm{OH}$ bond length on the $\mathrm{OH}$ stretching quantum number as well as on isotopic substitution, the calculated equilibrium value is increased based on experimental data for the rotational constant of the hydroxyl radical.[67,68] This is detailed in Table S4 in the ESI.

\section{Results and Discussion}

\subsection{Spectra of Monomeric $\alpha$-Fenchol}

We explored FTIR jet spectra of $\alpha$-fenchol before in the context of chirality recognition with $\alpha$-pinene[69]. They show in the high-wavenumber part of the $\mathrm{OH}$ stretching region two bands at 3675 and $3666 \mathrm{~cm}^{-1}$, with the latter having a higher intensity (Figure 3 ). This is in apparent agreement with the prediction of at least two of the conformers $(g-$ and $g+)$ being close in energy and separated by $8-9 \mathrm{~cm}^{-1}$ in their harmonic $\mathrm{OH}$ stretching wavenumbers (Table 1). However, more detailed analysis reveals that this match is superficial. The $g-$ conformer is slightly but almost consistently predicted to be the lowest in energy (in agreement with the conclusion from microwave spectroscopy[46,47]) and 


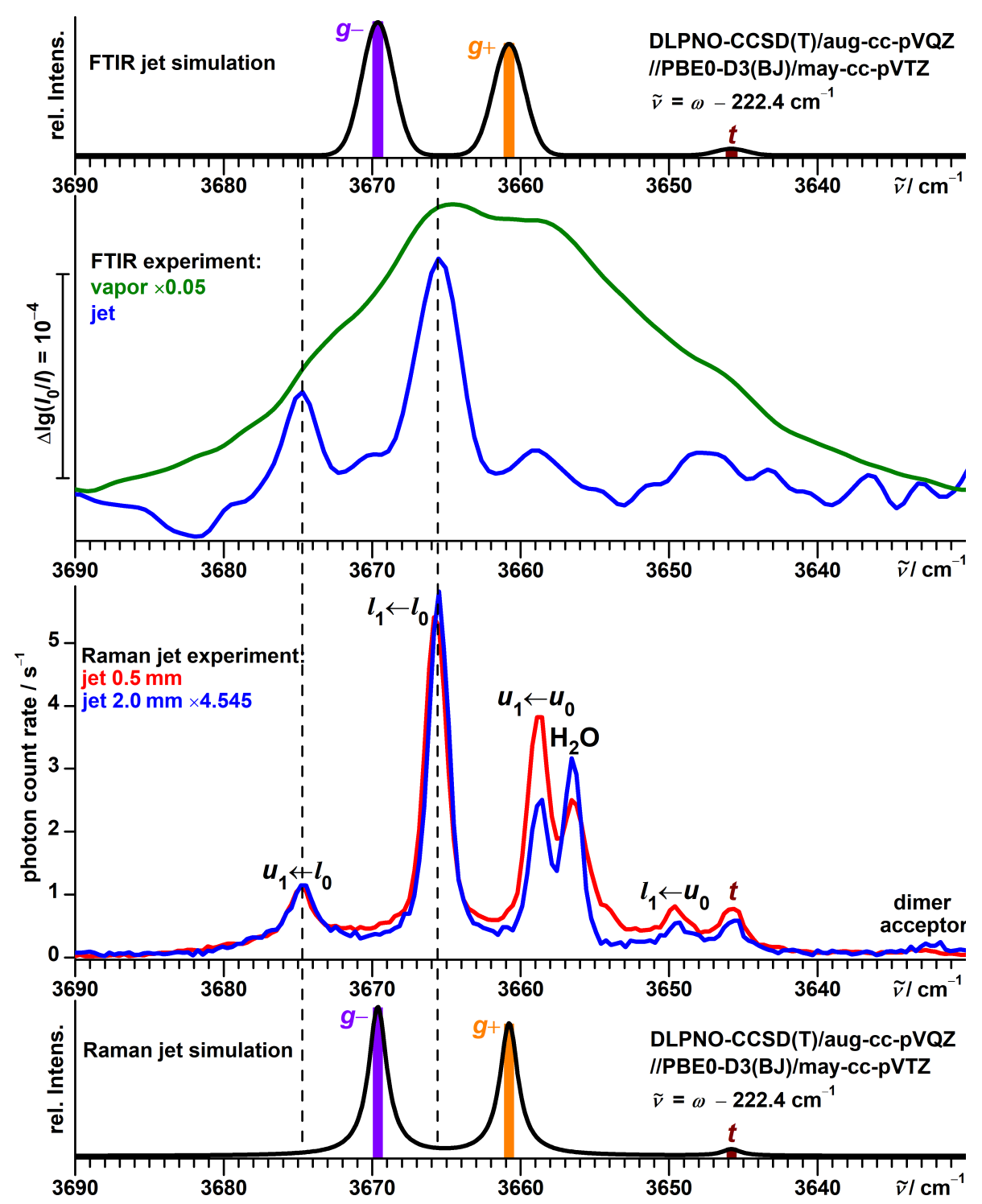

Figure 3. Top half: Comparison between simulated and experimental FTIR jet spectra of $\alpha$-fenchol. Also shown is an FTIR spectrum of the vapor at ambient temperature. Bottom half: Comparison between simulated and experimental Raman jet spectra of $\alpha$-fenchol at two different detection distances from the nozzle. For the simulations a Boltzmann distribution of localized conformers is assumed at a conformational temperature of $100 \mathrm{~K}$. Harmonic $\mathrm{OH}$ stretching wavenumbers are uniformly shifted according to the model of Ref. 48.

to feature the highest $\mathrm{OH}$ stretching wavenumber, which is in conflict with the observed sequence. The IR band strengths of these two conformers are predicted to be almost equal at all employed levels of theory and therefore cannot explain this discrepancy, either. Furthermore, the absolute band positions are not in good agreement with the predictions from a recently proposed model based on harmonic PBE0 calculations[48]. The model captures the $\mathrm{OH}$ stretching wavenumbers of 46 alcohols conformers within $\pm 3 \mathrm{~cm}^{-1}$ including those of borneol and isopinocampheol. For $\alpha$-fenchol, however, the errors would reach about $5 \mathrm{~cm}^{-1}$ for both conformers (simulation in the upper part of Figure 3) and would further escalate if the assignment was reversed. Finally, the FTIR spectrum of the vapor at ambient temperature has a spectral center of gravity notably shifted to lower wavenumbers relative to the jet. This can only partially be explained by the expected presence of the $t$ conformer at a lower wavenumber, as it has a predicted high relative energy at most levels of theory (Table 1) and, more importantly, an IR band strength only about half as 
Table 1: Differences between $\alpha$-fenchol conformers relative to $g$ - for harmonically zeropoint corrected energies and harmonic $\mathrm{OH}$ stretching wavenumbers at different computational levels. For values in parentheses the electronic contributions to the energy difference were replaced with DLPNO-CCSD(T)/aug-cc-pVQZ single-point values calculated on the respective geometries. A systematic underestimation of $\mathrm{OH}$ stretching wavenumber differences between $g$ and $t$ conformers at MP2 level was reported before[48].

\begin{tabular}{llccc}
\hline method & $\begin{array}{c}E_{0}(g+) \\
/ \mathrm{kJ} \mathrm{mol}\end{array}$ & $\begin{array}{c}\omega_{\mathrm{OH}}(g+) \\
/ \mathrm{cm}^{-1}\end{array}$ & $\begin{array}{c}E_{0}(t) \\
/ \mathrm{kJ} \mathrm{mol}\end{array}$ & $\begin{array}{c}\omega_{\mathrm{OH}}(t) \\
/ \mathrm{cm}^{-1}\end{array}$ \\
\hline MP2/6-311++G(d,p) & $0.04(-0.04)$ & -8 & $2.71(1.61)$ & -3 \\
PBE0-D3(BJ)/may-cc-pVTZ & $0.28(0.14)$ & -9 & $0.37(1.43)$ & -24 \\
B3LYP-D3(BJ)/may-cc-pVTZ & $0.33(0.12)$ & -8 & $0.81(1.52)$ & -17 \\
B2PLYP-D3(BJ)/may-cc-pVTZ & $0.22(0.07)$ & -8 & $1.51(1.54)$ & -12 \\
\hline
\end{tabular}

large. This is confirmed by comparison with the FTIR spectra of borneol (Figure S2) and isopinocampheol (Figure S5) which feature $t$ conformers with similar calculated properties (Figures S1 and S4).

The Raman jet spectra (lower half of Figure 3) help to unravel this mystery by revealing five additional bands in this spectral region. This is thanks to superior resolution and sensitivity of the Raman jet detection for alcohol monomer investigations, as well as higher conformational temperatures due to exclusive detection closer to the nozzle enabled by the narrow laser beam. One of these bands, at $3657 \mathrm{~cm}^{-1}$, reflects a downside of the method for this purpose, though, as it is caused by the mostly Raman-active symmetric stretching mode of a common water impurity which might obstruct alcohol signals. The two signals from the FTIR jet spectra are confirmed and retain their relative intensities upon variation of the detection distance. Three newly detected bands at 3659,3650 and $3646 \mathrm{~cm}^{-1}$ in contrast lose roughly half of their relative intensity at $2.0 \mathrm{~mm}$ distance compared to $0.5 \mathrm{~mm}$, while a very weak band at $3633 \mathrm{~cm}^{-1}$ instead gains relative intensity.

So how to explain the presence of six bands for $\alpha$-fenchol, when for borneol and isopinocampheol only a single band for each of three conformers was observed[48]? One possibility to consider are other impurities beyond water. Both microwave investigations reported $\beta$-fenchol as a minor component in the commercial samples of $\alpha$-fenchol with spectral intensities two orders of magnitude smaller[46,47]. The purity stated by the manufacturer for the sample used in the present work is $96.8 \%$. The harmonic $\mathrm{OH}$ stretching wavenumbers for the analogous conformers of $\alpha$ - and $\beta$-fenchol are predicted to be within $2 \mathrm{~cm}^{-1}$ or less (Figure S7). Any possible spectral contribution of $\beta$-fenchol is therefore expected to be too minor and too similar to be responsible for any of the additional signals (Figure S8).

Not technically impurities, but other species to consider, are dimers of $\alpha$-fenchol. They are expected to feature a dangling hydroxy group for the acceptor molecule of the hydrogen bond, typically scattering slightly downshifted from the monomer signals, as it is known from other saturated alcohols[70-72]. As aggregation builds up in the colder part of the expansion more distant from the nozzle, this is a plausible assignment for the weak signal at $3632 \mathrm{~cm}^{-1}$ gaining intensity. This assignment as well as the strongly downshifted donor mode will be discussed further below in section 3.8. For borneol and isopinocampheol no dimer signals (neither acceptor nor donor) could be observed, as their vapor pressures are an order of magnitude smaller compared to $\alpha$-fenchol.

This leaves five signals for assignment. One hint comes from a pattern of repeating spectral differences between four of these bands, highlighted in the center of Figure 4. A possible interpretation is the four energy level scheme shown in the same figure, in which spectral separations are translated into ground and excited state splittings with the method of combination differences. Such an assignment also has the beauty of explaining the observed changes in relative intensities. If the two high-wavenumber signals at 3675 and $3666 \mathrm{~cm}^{-1}$ do not originate from different conformers, but from the same state, it becomes 
understandable why their relative intensities are unaffected by changes in the experimental conditions. Likewise, the two signals at 3659 and $3650 \mathrm{~cm}^{-1}$ lose relative intensity at the same rate under colder conditions if they arise from the same excited state.

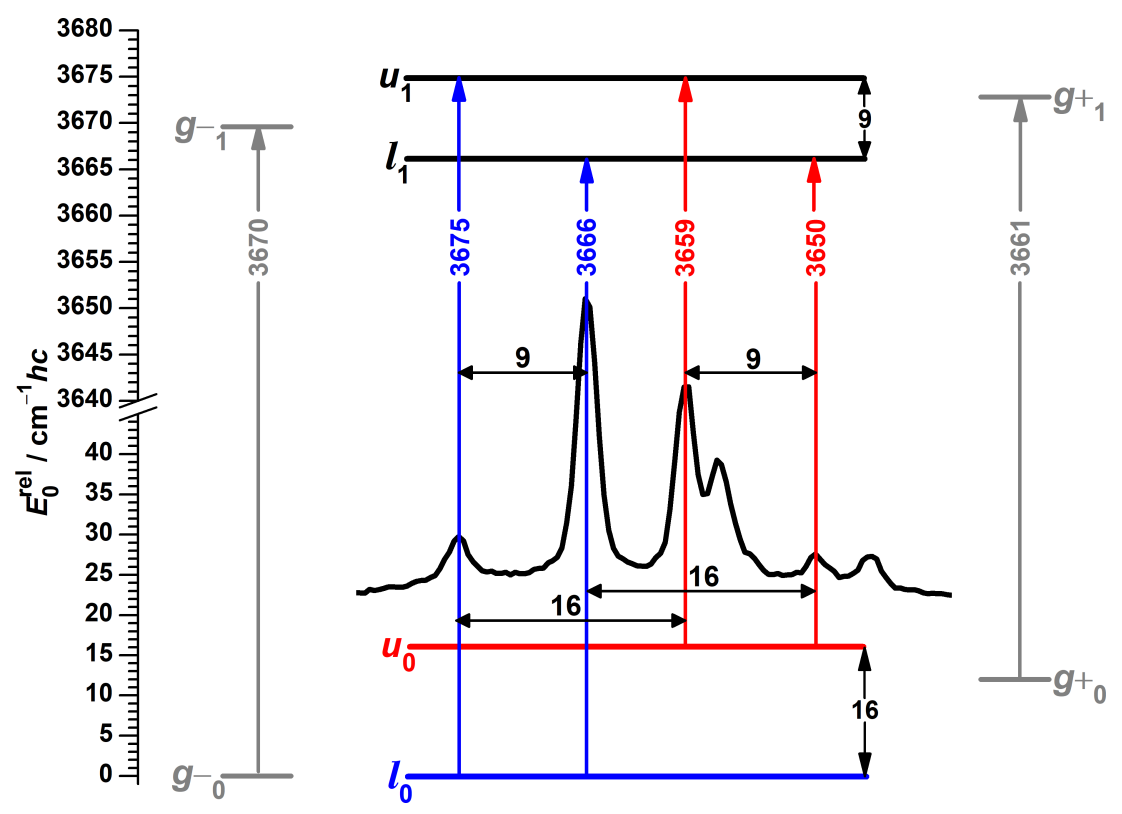

Figure 4. Raman spectral pattern in the $\mathrm{OH}$ stretching spectrum of $\alpha$-fenchol with repeating separations (black horizontal double-headed arrows) translated into torsional splittings (black vertical double-headed arrows). The ground state $l_{0}$ and transitions originating from it are colored blue, for the first excited torsional state $u_{0}$ likewise red, for hypothetical localized $g-$ and $g+$ states gray, the latter based on DLPNO-CCSD(T)/aug-cc-pVQZ//PBE0-D3(BJ)/may-cc-pVTZ calculations with shifted harmonic $\mathrm{OH}$ stretching wavenumbers[48]. $l$ stands for lower and $u$ for upper torsional state, the index 0 or 1 indicates the $\mathrm{OH}$ stretch quantum number.

A possible mechanism to explain the presence of additional bands in the spectrum of $\alpha$-fenchol, and their absence in those of borneol and isopinocampheol, is a tunneling interaction between the two $g$ conformers in the case of a near-degeneracy, leading to delocalization of the hydroxy hydrogen atom in the $\mathrm{OH}$ stretching ground and/or the excited state. Such an effect would also increase the energy difference between the torsional states but in first-order approximation not shift the centers of gravity for states and transitions. Indeed, the wavenumber average of these four bands $\left(3662.5 \mathrm{~cm}^{-1}\right)$ is in better agreement with the prediction for the average of the localized $g$ conformers according to the model based on PBE0 (3665.2 $\mathrm{cm}^{-1}$ ).[48] However, the required accidental energy near-degeneracy between non-equivalent conformations in their vibrational ground and/or $\mathrm{OH}$ stretch excited states is far from a routine observation, in particular for non-isotopologic symmetry breaking. The plausibility of this hypothesis will be investigated computationally in more detail in section 3.3. The final band at $3646 \mathrm{~cm}^{-1}$ can be assigned to the high-energetic and therefore localized $t$ conformer, in excellent agreement with its predicted position of $3645.8 \mathrm{~cm}^{-1}$.

As an attempt to localize the hydrogen atom also in the $g$ potential wells, and therefore simplify the spectrum, we deuterated the hydroxy group. Indeed, deuteration has a substantial impact on the spectral pattern, but different than anticipated by us, as the intensity becomes more evenly distributed between the same number of bands rather than collapsing into classically expected transitions.

Broad bands at 2733 and $2723 \mathrm{~cm}^{-1}$ in the OD stretching region of the Raman spectrum (Figure 5) are already present before deuteration and can likely be attributed to some overtone or combination excitations not involving the hydroxy hydrogen. Other spectral activity is readily assigned to impurities: $\mathrm{HDO}$ [73] $\left(2723 \mathrm{~cm}^{-1}\right.$, coinciding with the scaffold overtone/combination band), $\mathrm{D}_{2} \mathrm{O}[73,74]\left(2671 \mathrm{~cm}^{-1}\right)$ and residues of the 


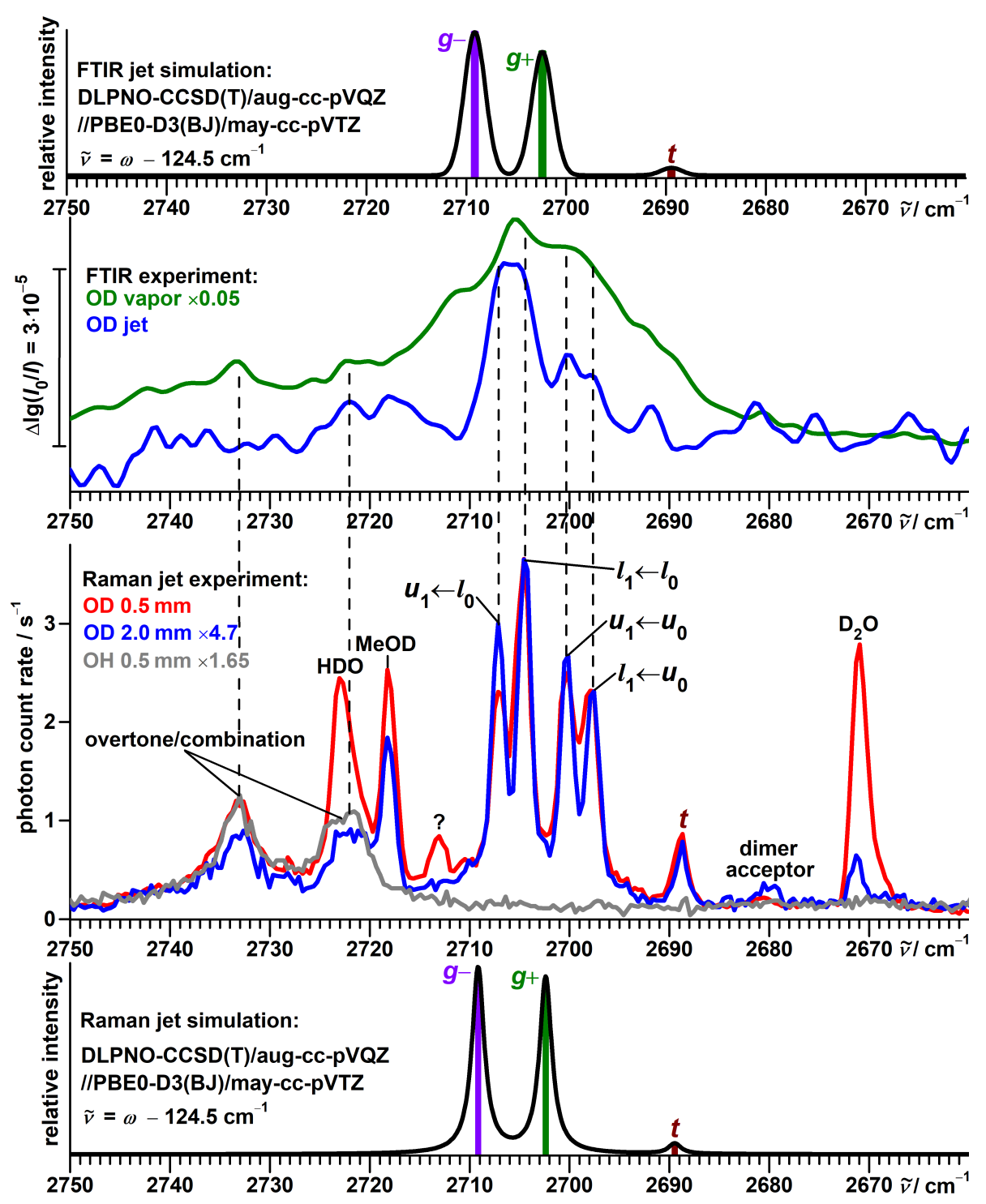

Figure 5. Top half: Comparison between simulated and experimental FTIR jet spectra of $\alpha$-fencholOD. Also shown is an FTIR spectrum of the vapor at ambient temperature, Bottom half: Comparison between simulated and experimental Raman jet spectra of $\alpha$-fenchol-OD at two different detection distances from the nozzle in the OD stretching region. Also shown is a spectrum of $\alpha$-fenchol-OH in this spectral region. For the simulations a Boltzmann distribution of localized conformers is assumed at a conformational temperature of $100 \mathrm{~K}$. Harmonic OD stretching wavenumbers are uniformly shifted according to the model of Ref. 48.

used deuteration reagent MeOD[75] $\left(2718 \mathrm{~cm}^{-1}\right)$. Eye-catching, due to again repeating separations, is a band pattern consisting of four components with similar intensities at 2707, 2704,2700 and $2697 \mathrm{~cm}^{-1}$. In analogy to the protiated alcohol, a possible interpretation is an assignment to a four-level scheme, as shown in Figure 6 with torsional splittings of 7 and $3 \mathrm{~cm}^{-1} h c$, reduced from 16 and $9 \mathrm{~cm}^{-1} h c$ by deuteration. The spectral center of gravity of $2702 \mathrm{~cm}^{-1}$ is slightly smaller than the predicted [48] average of $2705.8 \mathrm{~cm}^{-1}$ for the localized $g$ conformers, as for the protiated alcohol. The relative intensities within this band pattern change only very slightly upon increasing the detection distance from 0.5 to $2.0 \mathrm{~mm}$, which is consistent with a smaller torsional splitting of the OD stretch ground state as a reduced driving force for relaxation.

The FTIR jet spectrum (Figure 6), detected at a larger average distance from the nozzle, shows clear absorption for the unresolved 2707 and $2704 \mathrm{~cm}^{-1}$ transitions, while the 2700 


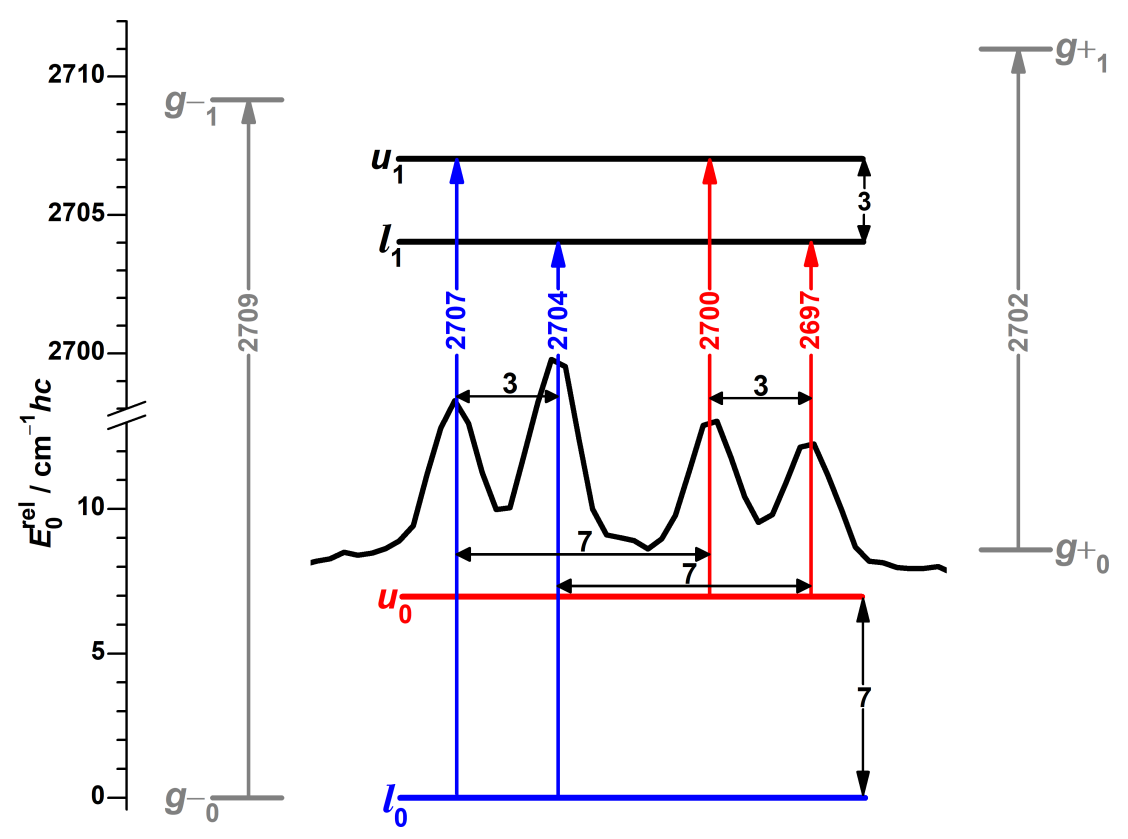

Figure 6. Raman spectral pattern in the OD stretching spectrum of $\alpha$-fenchol with repeating separations (black horizontal double-headed arrows) translated into torsional splittings (black vertical double-headed arrows). The ground state $l_{0}$ and transitions originating from it are colored blue, for the first excited torsional state $u_{0}$ likewise red, for hypothetical localized $g-$ and $g+$ states gray, the latter based on DLPNO-CCSD(T)/aug-cc-pVQZ//PBE0-D3(BJ)/may-cc-pVTZ calculations with shifted harmonic OD stretching wavenumbers[48]. $l$ stands for lower and $u$ for torsional upper state, the index 0 or 1 indicates the OD stretch quantum number.

and $2697 \mathrm{~cm}^{-1}$ signals only barely exceed noise level. This intensity discrepancy might be explained by differences in population or/and IR band strength; the more even absorption of the vapor at ambient temperature suggests the former influence to be the dominant one. We therefore conclude that the splitting of $7 \mathrm{~cm}^{-1} h c$ applies to the OD stretch groundstate and the one of $3 \mathrm{~cm}^{-1} \mathrm{hc}$ to the excited state. This assignment is also supported by theoretical considerations, as will be elaborated in the section 3.3.

Strong parallels between this interpretation and the one for the hydrogen chloride dimer, summarized in the introduction, are apparent. This includes values of absolute splittings, their changes with both deuteration and stretching excitation, as well as the observation of additional transitions for slightly asymmetric species.

A less intense band at $2689 \mathrm{~cm}^{-1}$ gains relative intensity closer to the nozzle and its position is in excellent agreement with the model prediction $\left(2689.5 \mathrm{~cm}^{-1}\right)$ for the $t$ conformer. Based on the opposite distance dependence of its relative intensity, the $2680 \mathrm{~cm}^{-1}$ signal is assigned to the acceptor of the most stable isomer of the deuterated dimer of $\alpha$-fenchol. A weak signal at $2713 \mathrm{~cm}^{-1}$ is only detected very close to the nozzle. This and the up-shifted position might hint at an origin from an even higher torsional state, similar to the situation for ethanol[71,76] and methanol[76]. No counterpart is observed for $\alpha$-fenchol-OH, though.

An interesting question is: Why were transitions from the second conformer (or more accurately: torsional state) not observed in the microwave investigations [46,47], despite the high detection sensitivity of this technique and low energy differences of only $16(\mathrm{OH})$ and $7 \mathrm{~cm}^{-1} h c(\mathrm{OD})$, equivalent to less than 0.2 and $0.1 \mathrm{~kJ} \mathrm{~mol}^{-1}$ ? For other tunneling systems very efficient relaxation between the split states was concluded, approaching or reaching effective temperatures estimated in the single-digit Kelvin range[32,35,36,48,77]. Notably, no splitting from internal rotation of the hydroxy group was reported in a neon jet investigation of but-2-yn-1-ol[78], despite it featuring a calculated barrier of similar height to prop-2-yn-1-ol (propargyl alcohol), for which a second set of transitions was observed 
at ambient temperature[79,80] and in an argon jet[81]. We infer that when using a strong relaxant agent[82] at a high pressure, such as neon at 2.0 or 4.5 bar in the former studies of $\alpha$-fenchol, and a large average detection distance from the nozzle, as typical for microwave spectroscopy, there is the possibility that the depopulation of the upper tunneling state might become too extensive for its observation. This issue was reported as well in the IR laser investigation of the $\mathrm{HCl}$ and $\mathrm{DCl}$ dimer and resolved by a switch from neon to argon and to a lower carrier gas pressure[35,36].

\subsection{Consistency Checks for Isotope Effects}

With simple models it is possible to check whether the assignments made for protiated and deuterated $\alpha$-fenchol are consistent with each other as well as with other alcohols.

Using a simple Morse oscillator model[83], the isotope effect on the ratio of the fundamentals can be expressed as:

$$
\frac{\tilde{v}_{\mathrm{i}}}{\tilde{v}_{0}}=\frac{\sqrt{r} \omega_{\mathrm{e}}-r 2 \omega_{\mathrm{e}} x_{\mathrm{e}}}{\omega_{\mathrm{e}}-2 \omega_{\mathrm{e}} x_{\mathrm{e}}}
$$

with $\tilde{v}_{\mathrm{i}}$ and $\tilde{v}_{0}$ being the fundamental wavenumbers of the isotopically modified and the unmodified molecule, respectively, $r$ the ratio of their reduced masses $\mu_{0} / \mu_{\mathrm{i}}, \omega_{\mathrm{e}}$ the harmonic wavenumber and $\omega_{\mathrm{e}} x_{\mathrm{e}}$ the anharmonicity constant of the unmodified molecule. If harmonic wavenumbers, mass ratios and anharmonicities do not differ too much, determined ratios of fundamental wavenumbers may be transferred between chemically related molecules. This is empirically known and used by spectroscopists for eight decades as the "ratio rule"[84]. With the $\mathrm{OH}$ stretching oscillator being very localized and decoupled (OD somewhat less so) and its anharmonicity being apparently widely unaffected by conformation and substitution[48], the requirements should be especially favorable for (not or weakly hydrogen-bonded) alcohols. This is indicated by observed uniform ratios for conformers of ethanol[71] and 1-propanol[72], and is here generalized across different alcohols. Using approximate but constant values of $r=1 / 1.8868[71]$ and $2 \omega_{\mathrm{e}} x_{\mathrm{e}}=180 \mathrm{~cm}^{-1}$ (when also pragmatically including off-diagonal anharmonicity of about $10 \mathrm{~cm}^{-1}$ )[48], the calculated fundamental ratios differ in the relevant $\omega_{\mathrm{e}}$-range of about $3870-3800 \mathrm{~cm}^{-1}$ [48] only between 0.7377 and 0.7379 . Replacing with the average value introduces an expected error of only $\pm 0.3 \mathrm{~cm}^{-1}$ for this wavenumber range, provided that the assumptions are met.

The performance of this model is demonstrated in Figure 7 using a training set of available jet data (Table S3) spanning ranges of $53(\mathrm{OH})$ and $40 \mathrm{~cm}^{-1}(\mathrm{OD})$ for 10 conformers of 5 alcohol monomers as well as acceptors of 6 alcohols dimers, which are only weakly perturbed by the hydrogen bond. The correlation is captured by the proposed simple one-parameter scaling model with a mean absolute error of $0.4 \mathrm{~cm}^{-1}$ and a maximum absolute error of $1 \mathrm{~cm}^{-1}$, which is equal to the experimental uncertainty of the data points. The isotope effect on the spectral positions assigned to the $g$ center of gravity, to the $t$ conformer and to the dimer acceptor of $\alpha$-fenchol fully meet the expectation, as evident from Figure 7.

Likewise, it has been shown that experimental tunneling splittings of protiated and deuterated alcohols (and also other series of compounds with hydrogen tunneling) can be directly correlated with each other as well, taking advantage of similar changes of the moment of inertia and the torsional wavenumber upon deuteration[85]. If the contributions to the assigned torsional splittings of $\alpha$-fenchol are dominated by tunneling, and less by asymmetry, the isotope effect should be roughly captured by this model as well. Indeed, this is the case for the splittings of both the $\mathrm{OH} / \mathrm{OD}$ stretch ground and excited states, as shown in Figure 8. With the data points for $\alpha$-fenchol being slightly above the regression line, this might indicate a larger impact of the asymmetry on the splitting of the deuterated isotopolog. 


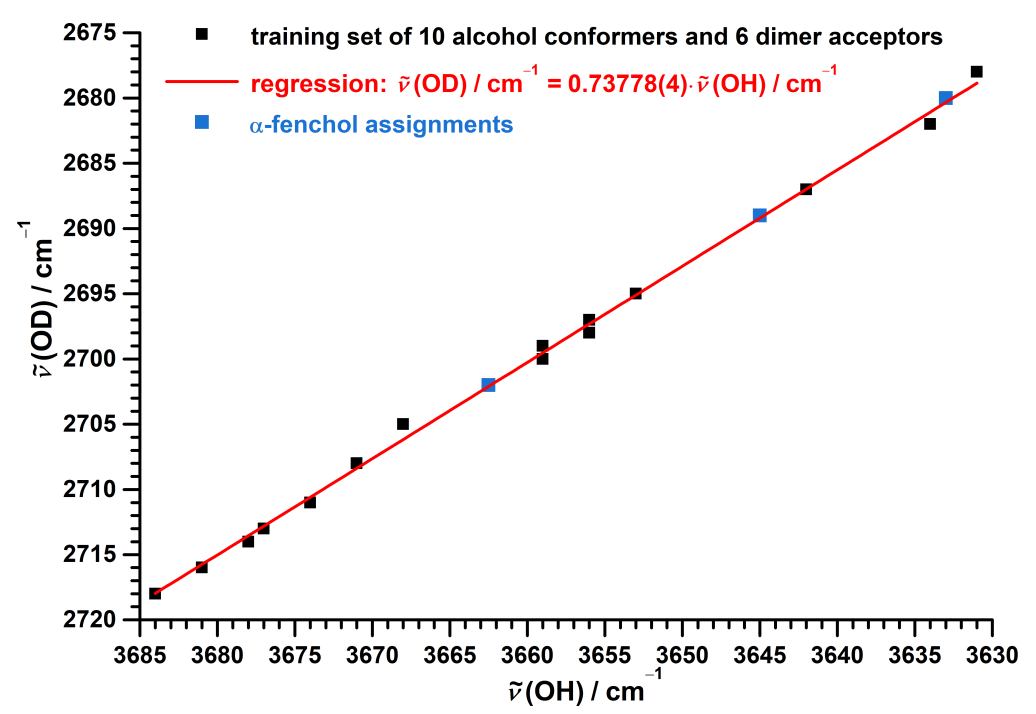

Figure 7. Correlation between experimental jet $\mathrm{OD}$ and $\mathrm{OH}$ stretching fundamental wavenumbers of alcohol monomers and alcohol dimer acceptors. Used data for the training set are listed in Table S3. The linear regression for the training set uses a fixed intercept of zero.

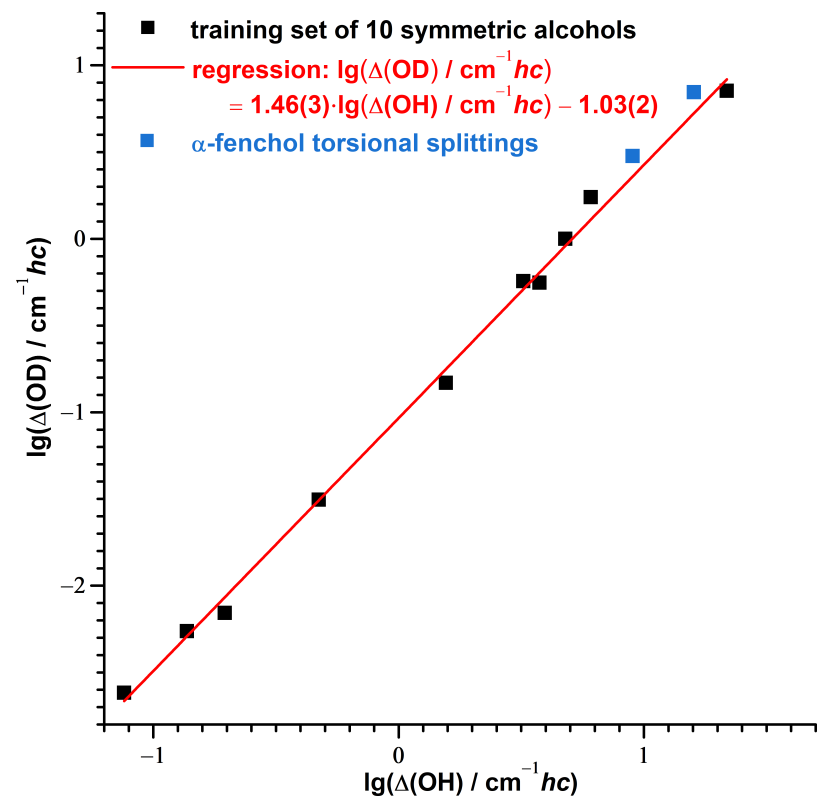

Figure 8. Correlation between the logarithmized experimental tunneling splittings $\lg \left(\Delta / \mathrm{cm}^{-1} h c\right)$ of deuterated and protiated symmetric alcohols (Direct Correlation Model of Ref. [85], details given there) compared with the torsional splittings assigned for the ground and the OH/OD stretch excited state of $\alpha$-fenchol.

\subsection{Theoretical Investigation of Torsional States of $\alpha$-Fenchol}

Unlike the systems mentioned in the introduction, there is no obvious symmetric parent for $\alpha$-fenchol to experimentally compare with. The closest open-chain analog is di-tert-butyl carbinol, which is however calculated to adopt as well an asymmetric conformation as a consequence of the steric repulsion between the bulky groups. Symmetric secondary alcohols with known tunneling splittings are 2-propanol[86], cyclohexanol[87] and cyclopropanol[88]. They feature experimental splittings at least an order of magnitude smaller, which correlate with calculated B3LYP-D3(BJ) barriers at least twice as high. In addition, the barrier of $\alpha$-fenchol is also narrower, with the $g$ equilibrium geometries calculated to be separated by $\Delta \tau(\mathrm{HCOH})=104^{\circ}$, while the width of the barrier amounts to at least $129^{\circ}$ for the other mentioned secondary alcohols. Besides the low energetic 
conformational difference and the characteristic isotope effect on the splittings, a particularly low and narrow barrier for $\alpha$-fenchol is therefore identified as a another piece of circumstantial evidence pointing towards the delocalization hypothesis.

To assess the torsional splitting, and the expected contribution from tunneling to it, we employ different computational models. As a first approach, it was demonstrated to be possible to reasonably estimate the tunneling splitting of symmetric alcohols only on the basis of calculated B3LYP properties of the enantiomeric $g$ geometries and the transition state in between[85]. To model the limiting case of the potential of $\alpha$-fenchol being perfectly symmetric, $g-/ g+$ averages are used for the barrier heights and harmonic torsional wavenumbers in these models. Calculated splittings are given in Table 2 and similar to the assigned ones $\left(16\right.$ and $\left.7 \mathrm{~cm}^{-1} h c\right)$.

Table 2: Predicted tunneling contributions $\Delta$ to the ground-state torsional splitting of $\alpha$-fenchol-OH and -OD according to different simple models[85] and the torsion 1D code based on B3LYP-D3(BJ)/may-cc-pVTZ calculations, in part with DLNPO-CCSD(T)/augcc-pVQZ corrections. For the simple models barrier heights and torsional wavenumbers were averaged between $g-$ and $g+$ (used values given in Table S6), for the torsion 1D code the torsional potential was either symmetrized by averaging both half-potentials or an additional barrier at the $g-/ g+$ transition state was added to compare with the localized scenario.

\begin{tabular}{llcc}
\hline Computational Level & Model & $\begin{array}{c}\Delta_{\mathrm{OH}} / \\
\mathrm{cm}^{-1} h c\end{array}$ & $\begin{array}{c}\Delta_{\mathrm{OD}} / \\
\mathrm{cm}^{-1} h c\end{array}$ \\
\hline B3LYP & Eckart Barrier[85] & 16.2 & 3.6 \\
B3LYP & Barrier Height[85] & 11.1 & 5.3 \\
B3LYP & Restricted Barrier Height[85] & 15.4 & 5.1 \\
B3LYP & Effective Barrier Height[85] & 17.8 & 7.3 \\
B3LYP & torsion 1D code symmetrization & 15.4 & 3.4 \\
DLNPO-CCSD(T)//B3LYP & torsion 1D code symmetrization & 15.9 & 3.5 \\
DLNPO-CCSD(T)//B3LYP & torsion 1D code localization & 14.4 & 2.6 \\
\hline
\end{tabular}

As an alternative, we solve the Schrödinger equation explicitly for a relaxed scan of the torsional potential. To validate the performance of the torsion $1 \mathrm{D}$ code in combination with the electronic B3LYP potential, we compare results with the experimental benchmarking data set for tunneling splittings for 27 symmetric alcohol species compiled in Ref. 85 . The correlation is shown in Figure 9. The experimental values are reproduced with a mean symmetric deviation factor MSDF (defined in Equation (2)) of 1.31 and a maximum symmetric deviation factor of 2.4. This means the model is on average a factor of 1.31 off-target in either direction.

$$
\mathrm{MSDF}=\frac{1}{n} \sum_{i}^{n} \exp \left|\ln \left(\frac{\Delta_{i}(\exp )}{\Delta_{i}(\text { calc })}\right)\right|
$$

Considering that the splittings span a range of four orders of magnitude, this is a very satisfactory performance for a one-dimensional relaxed DFT-based model. It is somewhat better than the best performing simple model of Ref. 85 (Effective Barrier Height Model, MSDF of 1.39, based on the same computational level and benchmarking set) and requires in contrast no adjusted parameters but more computations.

To assess again the symmetric limiting case, the potential of $(+)-\alpha$-fenchol is symmetrized by averaging the $180^{\circ}$ half-potentials separated at the $g-/ g+$ transition state (Figures S27 and S28). For the moment of inertia the value of the $g$ - conformer is used. The calculated moment of inertia varies only in a narrow corridor of less than $\pm 1 \%$ between the stationary points (Table S6), justifying the approximation of using a moment of inertia not depending on the torsional coordinate. The results, given in Table 2, are similar to both the estimations from the simpler models and the experimentally derived torsional splittings, supporting substantial contributions from the tunneling interaction to the latter. 


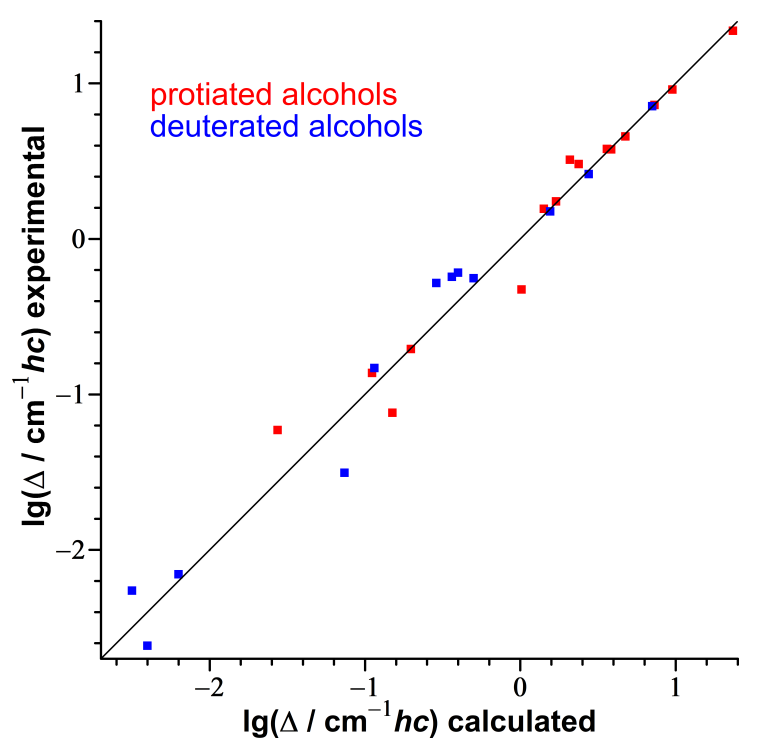

Figure 9. Correlation between logarithmized experimental tunneling splittings and calculated ones with the 1D torsion code based on electronic B3LYP-D3(BJ)/may-cc-pVTZ potentials for 27 isotopologs of 16 symmetric alcohols. The diagonal line represents perfect agreement. Data points are given in Table S5, diagrams with torsional potentials, energy levels, probability distributions and moment of inertia for all species are available in Figures S9-S25.

The calculated torsional splittings for the unmodified asymmetric B3LYP potential are $25 \mathrm{~cm}^{-1} h c(\mathrm{OH})$ and $19 \mathrm{~cm}^{-1} h c(\mathrm{OD})$ (Figure S26), larger than the observed ones of 16 and $7 \mathrm{~cm}^{-1} \mathrm{hc}$. While the calculated splittings are insensitive to small errors in the barrier height in the order of a few $\mathrm{cm}^{-1} h c$, they are very sensitive to absolute errors of this size for the asymmetry. The conformational energy difference is likely overestimated by the DFT methods (Table 1). Applying DLPNO-CCSD(T)/aug-cc-pVQZ single-point corrections to the six stationary points (minima and transitions states) and linearly scaling the connecting potential parts to match, results in the potential shown in Figure 10. The calculated splittings drop to $17.0(\mathrm{OH})$ and $5.3 \mathrm{~cm}^{-1} h c(\mathrm{OD})$, in good agreement with experiment.

In a simple perturbation model the tunneling splitting in the (hypothetical) symmetric case $\Delta$ and the energetic asymmetry between hypothetical localized states $\delta$ contribute to the observed total splitting $T$ according to Equation (3) $[4,5,34,35]$.

$$
T=\sqrt{\Delta^{2}+\delta^{2}}
$$

To separate the two contributions, artificial localization is applied to the DLPNOCCSD(T)//B3LYP-D3(BJ) potential by adding an additional narrow $\left(1^{\circ}\right)$ but towering $\left(10^{6} \mathrm{~cm}^{-1} h c\right)$ rectangle barrier at the $g-/ g+$ transition state which suppresses tunneling (Figure S32). This lowers the splitting of the first two torsional states from $T=17.0 \mathrm{~cm}^{-1} \mathrm{hc}$ to $\delta=9.1 \mathrm{~cm}^{-1} h c$, which in turn yields $\Delta=14.4 \mathrm{~cm}^{-1} h c$ according to Equation (3), in good agreement with the results from the other approaches (Table 2). For the deuterated alcohol artificial localization converts $T=5.3 \mathrm{~cm}^{-1} h c$ into $\delta=4.6 \mathrm{~cm}^{-1} h c$, yielding $\Delta=2.6 \mathrm{~cm}^{-1} h c$, again in decent agreement with the other estimates. With $\Delta>\delta$ the protiated alcohol is strongly delocalized, while the deuterated is moderately, in line with integrated probability distribution shares in the $g-/ g+$ potential wells of $69 \% / 31 \%(\mathrm{OH})$ and $88 \% / 12 \%(\mathrm{OD})$ (Figure 10). To reproduce the same $T$ in the absence of tunneling, the $g+$ well would have to be lifted by $9.5 \mathrm{~cm}^{-1}$ for $\mathrm{OH}$ and by $0.8 \mathrm{~cm}^{-1}$ for OD in the same way in which DLPNO refinements were introduced before, without changing the other stationary points. Small deviations of these values from the term $T-\delta$ accrue from the different curvatures of the potential wells, which can lead to further interesting effects, as described below. 

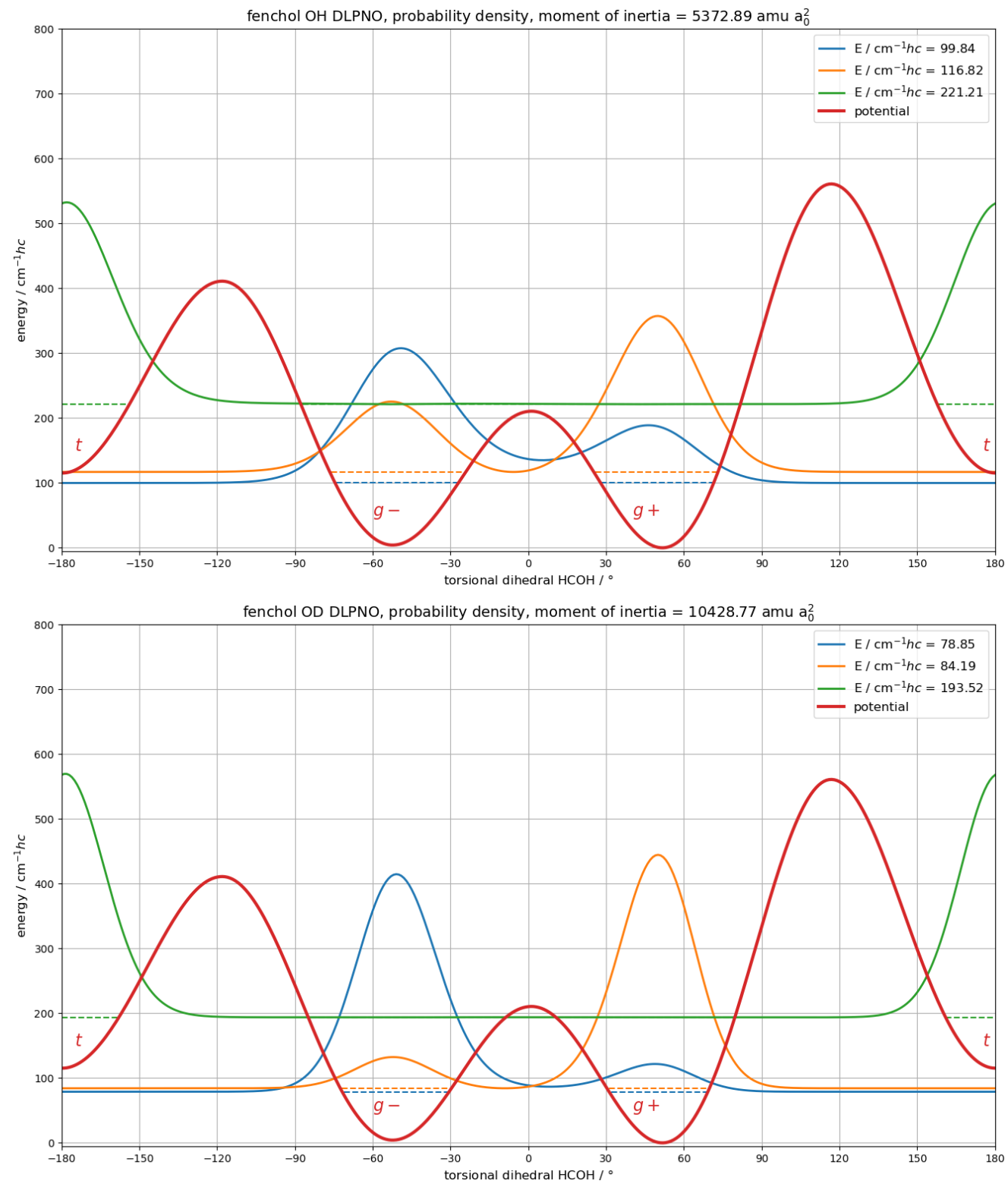

Figure 10. Electronic torsional potential (red trace) of (+)- $\alpha$-fenchol calculated at B3LYP-D3(BJ)/maycc-pVTZ level and scaled to DLPNO-CCSD(T)/aug-cc-pVQZ single-point corrections for the six stationary points. A constant moment of inertia, as calculated for the $g$ - minimum geometry, was used. For the protiated (top) and the deuterated alcohol (bottom) the two lowest torsional states (blue and orange horizontal dashed traces) are separated by $17.0(\mathrm{OH})$ and $5.3 \mathrm{~cm}^{-1} \mathrm{hc}(\mathrm{OD})$ and their probability densities (blue and orange solid traces) are strongly $(\mathrm{OH}: 69 \% / 31 \%)$ to moderately (OD: $88 \% / 12 \%$ ) delocalized between the $g-/ g+$ potential wells. In contrast, for the third torsional state the probability density (green solid trace) is very strongly localized ( $>99 \%)$ in the $t$ potential well.

At the DLPNO-CCSD(T) single-point level the electronic energy of the $g+$ equilibrium geometry is slightly lower than the one for $g-$. This sequence is inverted when the respective full harmonic zero-point correction, calculated at DFT level, is added. The same inversion is observed when considering the anharmonic torsional energy in the one-dimensional DLPNO-CCSD(T)/ / B3LYP-D3(BJ) potential, where the ground-state has a larger probability density in the shallower $g$ - well (Figure 10), in agreement with the conclusion from microwave spectroscopy[47]. The different local zero-point energies enable also the possibility of scenarios in which the asymmetry between the localized torsional states is smaller or/and inverted for deuterated $\alpha$-fenchol. This means the deuterium atom 
might be more strongly delocalized than the protium atom or/and might have a higher integrated probability density in a different potential well. Isotopomeric conformational change[89] and isotopomeric polymorphism[90] upon hydroxy group deuteration are not unprecedented, but so far only documented for hydrogen-bonded systems. As explored in the ESI in Figure S35, the window for such an occurrence is small but within the uncertainties of the calculations. The assignment of the main isotopolog based on the substitution geometry and quadrupole constants of the deuterated compound [47] might thus not be entirely reliable.

If not overcompensated by a higher asymmetry, a decrease of the splitting is expected upon $\mathrm{OH} / \mathrm{OD}$ stretch excitation. This is caused by increases of both the adiabatic barrier (higher $\mathrm{OH} / \mathrm{OD}$ stretching wavenumber at the transition state) and the moment of inertia (elongation of the vibrationally averaged bond) acting as the effective tunneling mass. Because differences in $\mathrm{OH}$ stretch fundamental wavenumbers are very similar to differences in B3LYP harmonic wavenumbers, at least for the minima[48], we estimate the torsional potential for the $\mathrm{OH}$ stretch excited state by adding the harmonic wavenumbers to the DLPNO-CCSD $(\mathrm{T})$ electronic energies of the stationary points and again linearly scaling the connecting potential parts. This assumes that changes to the shape of the potential, i.e., torsional positions of stationary points, can be neglected. The deflection of the $\mathrm{OH}$ or $\mathrm{OD}$ bond length upon vibrational excitation is estimated by the change of the rotational constants of the hydroxy radical[67,68], as detailed in Table S4. This simple model captures the available experimental alcohol data for the reduction of splittings upon $\mathrm{OH}$ stretch excitation reasonably well for methanol (exp: $9.1 \rightarrow 6.3 \mathrm{~cm}^{-1} h \mathrm{c}$ [91], calc: $9.5 \rightarrow 7.7 \mathrm{~cm}^{-1} h c$ (Figure S38)) and propargyl alcohol (exp: $22 \rightarrow 18 \mathrm{~cm}^{-1} h c$ (section 3.4), calc: $20.4 \rightarrow 16.9 \mathrm{~cm}^{-1} h c$ (Figure S39)). The same is true for $(+)-\alpha$-fenchol for both the $\mathrm{OH}$ (exp: $16 \rightarrow 9 \mathrm{~cm}^{-1} h c$, calc: $17.0 \rightarrow 10.7 \mathrm{~cm}^{-1} h c$ ) and the OD species (exp: $7 \rightarrow 3 \mathrm{~cm}^{-1} h c$, calc: $\left.5.3 \rightarrow 3.0 \mathrm{~cm}^{-1} h c\right)$, with details for the excited states given in Figure S30.

The hypothetical localized $g$ - conformer is calculated to feature a lower energy but higher $\mathrm{OH}$ and $\mathrm{OD}$ stretching wavenumbers than the $g+$ conformer. These differences are of similar size, so that the excitation is expected to reduce the asymmetry for both the $\mathrm{OH}$ and OD species. Artificial localization (Figure S34) yields $\delta=1.6(\mathrm{OH})$ and $-1.7 \mathrm{~cm}^{-1} \mathrm{hc}$ (OD), meaning very close near-degeneracies. Interestingly, for the deuterated alcohol the sign of the asymmetry is inverted, so that a switch upon OD excitation from predominant $g-$ to $g+$ character for the torsional ground state is calculated. Equation 3 yields $\Delta=$ $10.5(\mathrm{OH})$ and $2.4 \mathrm{~cm}^{-1} h c(\mathrm{OD})$. With $\Delta \gg \delta(\mathrm{OH})$ and $\Delta>|\delta|(\mathrm{OD})$ both stretch-excited isotopologs feature strongly delocalized torsional wave functions - more so than in the respective ground state, despite the increases of both the barrier height and the moment of inertia from excitation.

As a first approach to evaluate relative transition intensities, torsional Franck-Condon factors (FCF, equation 4) can be analyzed, obtained by factorizing the dependence of the transition moment $M$ on the stretching wave function $\psi$ (being evaluated at a fixed torsional dihedral $\tau_{\mathrm{e}}$ and depending on the stretching quantum number $v$ and the stretching coordinate $r$ ) and the torsional wave function $\chi$ (depending on the stretching $v$ and the torsional quantum number $m$ as well as the torsional coordinate $\tau$ )(equation 5 ). This factorizing relies on the assumption that the dependencies of the dipole moment $\mu$ on the stretch and torsional coordinates are separable or the latter is negligible[92,93].

$$
\begin{aligned}
\mathrm{FCF} & =\left\langle\chi_{m^{\prime}}^{v^{\prime}}(\tau) \chi_{m}^{v}(\tau)\right\rangle^{2} \\
M^{2} & =\left\langle\psi_{v^{\prime}}^{\tau_{\mathrm{e}}}(r) \chi_{m^{\prime}}^{v^{\prime}}(\tau)|\mu(r, \tau)| \psi_{v}^{\tau_{\mathrm{e}}}(r) \chi_{m}^{v}(\tau)\right\rangle^{2} \approx\left\langle\psi_{v^{\prime}}^{\tau_{\mathrm{e}}}(r)|\mu(r)| \psi_{v}^{\tau_{\mathrm{e}}}(r)\right\rangle^{2} \cdot\left\langle\chi_{m^{\prime}}^{v^{\prime}}(\tau) \mid \chi_{m}^{v}(\tau)\right\rangle^{2}
\end{aligned}
$$

The ground and first excited torsional states have preponderantly, but not dominantly, $g-$ and $g+$ character, respectively, for protiated $(+)-\alpha$-fenchol in both the $\mathrm{OH}$ stretching 
ground and excited state. The medium-wavenumber transitions $l_{1} \leftarrow l_{0}$ and $u_{1} \leftarrow u_{0}$, connecting torsional wave functions with the same character and same number of nodes, have the largest FCFs. FCFs for the satellite $u_{1} \leftarrow l_{0}$ and $l_{1} \leftarrow u_{0}$ transitions are about $4 \%$ of this size (Table S7). This is about three times more than for borneol (Table S1) and about a hundred times more than for isopinocampheol (Table S2), but clearly less than the observed intensity ratios for $(+)-\alpha$-fenchol (Figure 4$)$. An error in the calculated asymmetry of the potential is likely not to blame for this deviation, as the agreement cannot be substantially improved by varying the asymmetry in a concerted way for the $\mathrm{OH}$ stretch ground and excited state (Figure S36). This underestimation of transition intensities connecting torsional wave function with different number of nodes by the Franck-Condon model is reminiscent of the situation for $\mathrm{OH}$ stretching spectra of symmetric hydroperoxides [93-95] It was explained by a neglected component of the transition dipole moment being an antisymmetric function of the torsional coordinate, which needs to be factored into the symmetry selection rules.

In contrast, for the deuterated alcohol the calculated FCF ratios for both $u_{1} \leftarrow l_{0}$ $/ l_{1} \leftarrow l_{0}$ and $u_{1} \leftarrow u_{0} / l_{1} \leftarrow u_{0}$ are close to unity (Table S8), in good agreement with the observed intensity ratios (Figure 6). These values can be understood by the OD torsional wave functions being mostly localized in different wells for the OD stretch ground and excited state, which reduces the partial cancelation between positive and negative contributions of $\chi_{m^{\prime}}^{v^{\prime}} \chi_{m}^{v}$ to the integral. This also lowers the expected impact of neglected sign changes of the transition dipole moment function.

Alternatively, one can also attempt to add the small variation of the non-torsional harmonic zero-point energy of the stationary points to the scaling of the potential. Considering the shortcomings of the harmonic approximation and $\alpha$-fenchol featuring several low-wavenumber normal modes, in part with multiple modes having some torsional character depending on the conformer, such an adiabatic separation is not necessarily an improvement over the purely electronic potential. This approach leads to similar splittings of $T=17.8(\mathrm{OH})$ and $10.0 \mathrm{~cm}^{-1} h c(\mathrm{OD})$ in the ground state as well as $8.5(\mathrm{OH})$ and $3.1 \mathrm{~cm}^{-1} h c(\mathrm{OD})$ in the $\mathrm{OH}$ and OD stretch excited state, with details given in Figures S29 and S31. A qualitative difference is that no longer a conformational inversion upon OD stretch excitation is predicted. This results in larger FCF ratios (more localized intensity) than for the protiated alcohol (Tables S9 and S10), contrary to the experimental change upon deuteration.

While both variants of the model reproduce the observed torsional splittings quite well, a more sophisticated future investigation of relative intensities is required to investigate whether the highly unusual OD stretching spectrum is indeed caused by a conformational inversion upon excitation, as suggested by the FCFs. This would probably require both a less approximated potential (with the relatively large molecular size of $\alpha$-fenchol $\left(\mathrm{C}_{10} \mathrm{H}_{18} \mathrm{O}\right)$ being one challenge) as well as explicit calculation of transition moments. The importance of the latter is further stressed by the result of the next section, where we show that there can be major differences between relative IR and Raman activities in the limiting case of a symmetric alcohol.

\subsection{Experimental Support from Monomeric Propargyl Alcohol}

To also compare experimentally with a fully symmetric alcohol, we investigate propargyl alcohol. While the structural similarity to $\alpha$-fenchol is limited (Figure 1), we still believe this species to be especially well suited for a number of reasons:

- The ground-state splitting is well determined by rotational spectroscopy (about $\left.22 \mathrm{~cm}^{-1} h c\right)[79,80]$ and of similar size to the one proposed here for $\alpha$-fenchol $\left(16 \mathrm{~cm}^{-1}\right.$ $h c)$.

- Propargyl alcohol has a relatively high volatility (normal boiling point $115^{\circ} \mathrm{C}$ ), which allows to access high vapor pressures without heating, which is helpful to retain signal intensity in mild expansions with low carrier gas pressure. 
- The $t$ conformer of propargyl alcohol, diastereomeric to the two enantiomeric $g$ conformers interacting through tunneling, is calculated to be very high in energy (about $7 \mathrm{~kJ} \mathrm{~mol}^{-1}$ ) and therefore does not perturb the spectrum of the $g$ conformers.

- The presence of the $\pi$-system leads to both hydroxy groups donating a hydrogen bond each in dimers of propargyl alcohol[96,97]. This shifts their OH stretching fundamentals out of the monomer spectral region, so that the latter can be analyzed without the risk of contamination from signals of dangling hydroxy groups of dimers. This conclusion for propargyl alcohol will be reconfirmed in section 3.5.

In previous work[48] we investigated Raman jet spectra of propargyl alcohol. Despite only the enantiomeric $g$ conformers being energetically relevant, we observed two signals in the monomer $\mathrm{OH}$ fundamental region at 3662 and $3658 \mathrm{~cm}^{-1}$ (Figure 11, violet trace). The weaker lower-wavenumber signal rapidly loses relative intensity in colder parts of the expansion more distant from the nozzle. We interpreted this spectral splitting of $4 \mathrm{~cm}^{-1}$ as a lowering of the tunneling splitting by this amount upon $\mathrm{OH}$ stretch excitation. The proposed energy level scheme based on these observations is shown in the right part of Figure 11, assigning Raman-active symmetry-conserving transitions $\left(l_{1} \leftarrow l_{0}\right.$ and $u_{1} \leftarrow u_{0}$ ). The derived $\mathrm{OH}$ stretching fundamental wavenumber of a hypothetical localized $g$ conformer $\left(3660 \mathrm{~cm}^{-1}\right)$ is in decent agreement with the predicted one by the model for shifting the harmonic PBE0 wavenumber $\left(3657 \mathrm{~cm}^{-1}\right)$ [48]. No Raman activity for conceivable symmetry-changing transitions $\left(u_{1} \leftarrow l_{0}\right.$ and $\left.l_{1} \leftarrow u_{0}\right)$ at 3680 or $3640 \mathrm{~cm}^{-1}$ was observed.

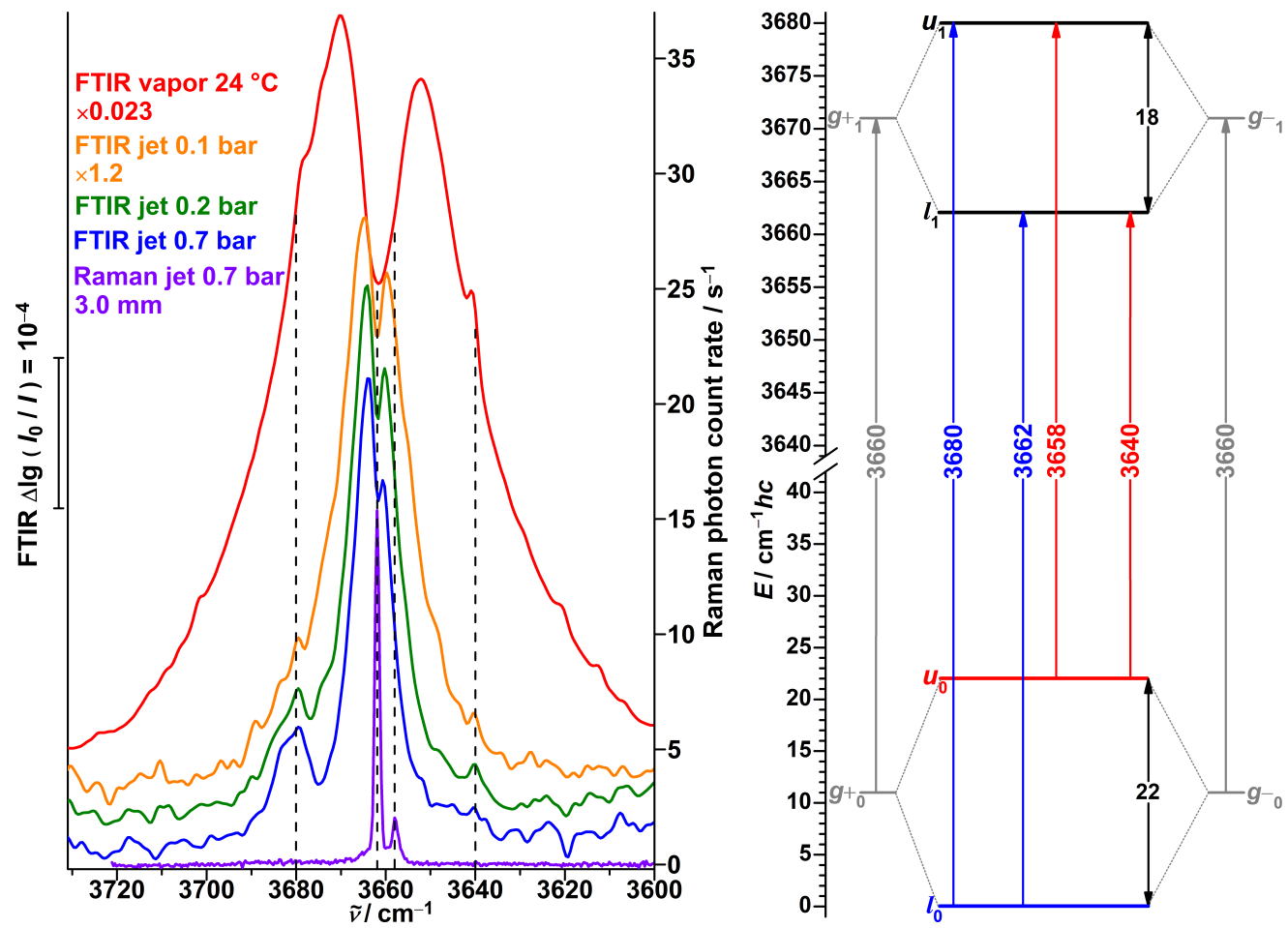

Figure 11. Left: FTIR jet and vapor spectra as well a Raman spectrum of propargyl alcohol in the $\mathrm{OH}$ stretching monomer spectral region. Assigned positions of band centers are indicated with dashed lines. Right: Energy level scheme with the ground state and transitions originating from it in blue, likewise for the upper tunneling state in red, as well as for hypothetical localized states in gray.

Analogous transitions for hydroperoxides were shown to have non-negligible infrared activity for multi-quanta $\mathrm{OH}$ stretching excitation[93-95], contrary to the symmetryselection rules from the simple vibrational Franck-Condon model; for single-quantum $\mathrm{OH}$ stretch excitation even intensities comparable with the symmetry-conserving transitions are predicted $[93,95]$. Considering the structural similarities between alkyl hydroperoxides and alcohols, we extend here our investigation of propargyl alcohol to FTIR detection. An 
early grating spectrometer study of propargyl alcohol vapor yielded a double-peak band centered around $3663 \mathrm{~cm}^{-1}$ [98]. A later high-resolution jet study reported a rich amount of transitions in the $3666-3660 \mathrm{~cm}^{-1}$ range, but did not explore beyond[99]. Using a lower resolution but a broader spectral range, we indeed observe a band at the expected position of $3680 \mathrm{~cm}^{-1}$ in the cold jet expansion (Figure 11, blue trace), at roughly one fifth of the intensity of the main band. In warmer expansions (green and orange traces), utilizing low carrier gas pressures, the signal is increasingly obstructed by the rovibrational R-branches of the symmetry-conserving transitions and converts to a shoulder in the spectrum of the stationary vapor at ambient temperature (red trace). At the second expected position at $3640 \mathrm{~cm}^{-1}$ a similar shoulder is observed. This second symmetry-changing transition is only weakly visible in the jet spectra, as the originating upper tunneling state is barely populated in a cold expansion and the signal is partially obstructed in the warmer expansions by the P-branches.

Satellites, shifted by +8 and $-9 \mathrm{~cm}^{-1}$ from the main band, with similar relative intensities were also observed for propargyl alcohol in an argon matrix but were not discussed[100]. Possible interpretations are that tunneling is quenched by the matrix environment and an unrelated site splitting is present, or that the tunneling splitting is reduced - the latter is observed, but far more drastically, for the propargyl alcohol $\cdots$ argon complex[81].

In conclusion, the FTIR jet spectra of propargyl alcohol confirm our previous interpretation of the Raman jet spectra as well as the expectation that symmetry-changing transitions do not follow the simple vibrational Franck-Condon scheme (Table S11) but carry nonnegligible IR activity $[93,95]$. The FTIR jet spectra of propargyl alcohol and $\alpha$-fenchol at cold conditions are very similar, both featuring one intense band and a higher-wavenumber less-intense band, separated by the torsional splitting of the excited $\mathrm{OH}$ stretching state. In contrast, the Raman jet spectra are quite different. The larger number of observed bands for $\alpha$-fenchol in part originate from the lower relative energy of the $t$ conformer and the dangling hydroxy group of its dimer. Furthermore, additional transitions between the torsional states carry Raman activity, while they do not measurably for propargyl alcohol. This might hint at a symmetry-based Raman selection rule, which is relaxed by the slight asymmetry present in $\alpha$-fenchol, or it may based on with specific IR and Raman transition moments in each molecular case. In any case, for the more strongly asymmetric borneol and isopinocampheol no Raman-activity for additional bands could be observed[48]), which is likely connected to negligible overlap between the strongly localized torsional wave functions (Figures S3 and S6). This highlights the special in-between status of the slightly asymmetric $\alpha$-fenchol, to which we attribute the observed spectral activity for additional transitions.

\subsection{Computational Results for Propargyl Alcohol Dimers}

To assign observed additional downshifted bands in the FTIR and Raman spectra and reconfirm that no dimer signal is expected to perturb the monomer region, we also investigated dimers of propargyl alcohol. Their conformational space was first explored by Mani and Arunan at MP2/6-311+G(3df,2p) level, who reported seven isomers[96], and later expanded to nine isomers by Saini and Viswanathan at M06-2X/6-311++G(d,p) level[97]. These nine isomers were here reoptimized at B3LYP-D3(BJ)/may-cc-pVTZ level and categorized. Building on the nomenclature established for structurally related dimers of 1-indanol[101], benzyl alcohol[15,102] and 1-phenylethanol[15], we first state the conformation of the constituting monomers gauche or trans in the sequence donor/acceptor, leading to the combinations $g g, g t, t g$ and $t$. This is followed by hom or het, describing whether the signs for the $\mathrm{HOC}_{\alpha} \mathrm{C}_{\beta}$ and $\mathrm{H}^{\prime} \mathrm{O}^{\prime} \mathrm{C}_{\alpha}^{\prime} \mathrm{C}_{\beta}^{\prime}$ dihedrals (in a $g g$ combination) or for the $\mathrm{HOC}_{\alpha} \mathrm{C}_{\beta}$ and the $\mathrm{E}^{\prime} \mathrm{O}^{\prime} \mathrm{C}_{\alpha}^{\prime} \mathrm{C}_{\beta}^{\prime}$ dihedrals (in a gt combination) agree or disagree with each other, with $\mathrm{E}$ standing for the coordinated oxygen electron pair. Finally, the acceptor types for established hydrogen bonds are stated, in the same sequence as the conformations of their monomer donors, with the four possibilities $\mathrm{O}^{g}, \mathrm{O}^{t}, \pi$ and $\mathrm{V} . \mathrm{O}^{g}$ and $\mathrm{O}^{t}$ state 
the qualitative arrangement of the $\mathrm{EOC}_{\alpha} \mathrm{C}_{\beta}$ dihedral for the coordinated oxygen electron pair, V stands for a 'void' acceptor, meaning a dangling hydroxy group. Going through these categorizations, it was noticed that within the nine previously reported isomers several conceivable combinations were not represented, which were subsequently explored. Furthermore, in both previous studies it was presupposed that energetically relevant isomers have to feature an $\mathrm{OH}$.. O hydrogen bond. However, for the dimers of 1-indanol, benzyl alcohol and 1-phenylethanol it has been found that isomers with two OH $\cdots \pi$ hydrogen bonds can be competitive enough to be observed in a jet expansion[15,101,102]. These considerations lead to five new isomers, among them are the third, fourth and sixth most stable overall. The six leading isomers are all composed of two gauche monomer conformers and feature two hydrogen bonds. They are the hom and het variants of the $\mathrm{O}^{t} \pi, \mathrm{O}^{g} \pi$ and $\pi \pi$ motifs and their structures are given in Figure 12 together with their $\mathrm{OH}$ stretching vibrational properties. The homochiral dimerization preference in an $\mathrm{O}^{g} \pi$ motif qualitatively parallels that for benzyl alcohol dimers[15]. The full list of isomers is available in Table S12.
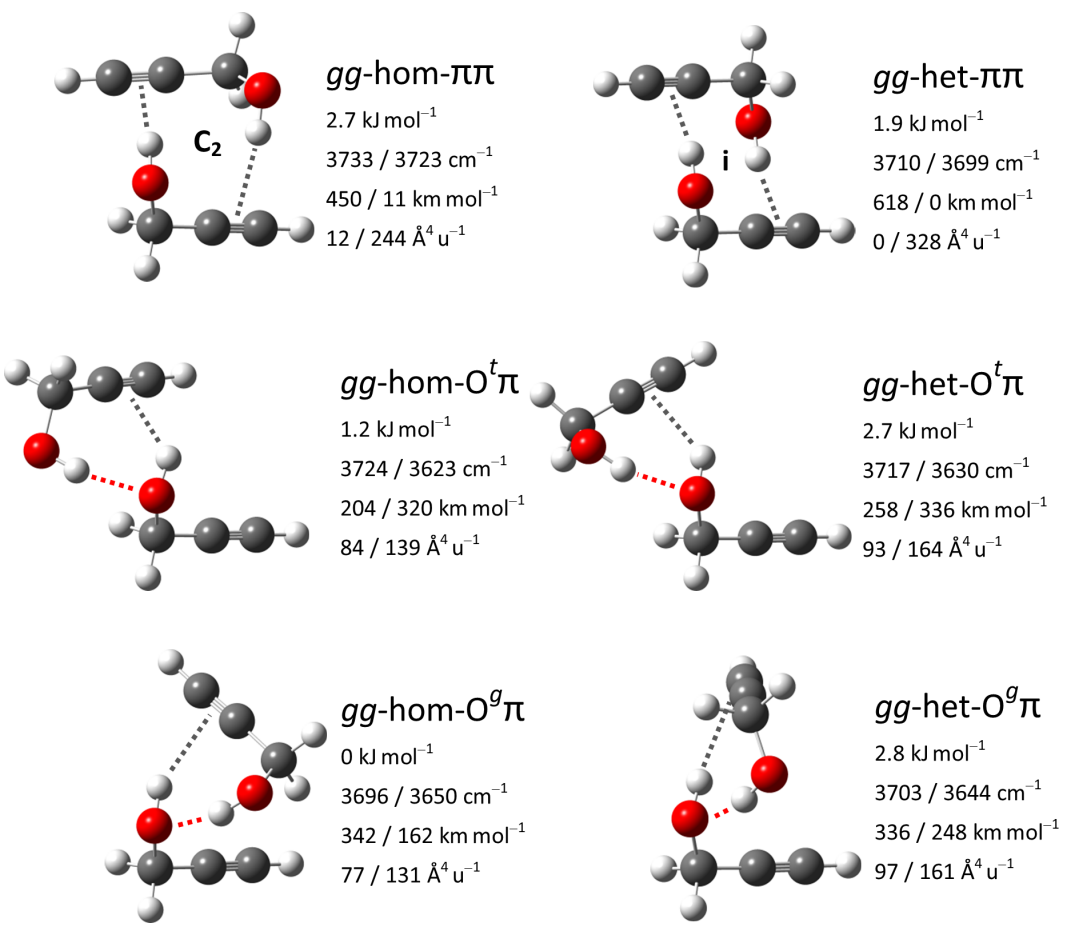

Figure 12. Leading propargyl alcohol dimer structures with harmonically zero-point-corrected relative energies, unscaled harmonic $\mathrm{OH}$ stretching wavenumbers as well as associated IR intensities and Raman activities at B3LYP-D3(BJ)/may-cc-pVTZ level. Indicated, where present, are symmetry elements as well as $\mathrm{OH} \cdots \mathrm{O}$ hydrogen bonds by red and $\mathrm{OH} \cdots \pi$ by black dotted lines.

The calculated vibrational properties promise complementary information from FTIR and Raman jet spectroscopy for the discrimination between the three relevant motifs. In the $\mathrm{O}^{t} \pi$ motif the two $\mathrm{OH}$ stretching oscillators are only weakly coupled and the more strongly downshifted fundamental localized in the $\mathrm{OH} \cdots \mathrm{O}$ hydrogen bond features both higher IR and Raman activity than the less downshifted fundamental for the $\mathrm{OH} \cdots \pi$ hydrogen bond. In contrast, in the $\mathrm{O}^{g} \pi$ motif the two oscillators are strongly coupled, with the out-of-phase-combination having higher IR activity and the in-phase-combination having higher Raman activity. Finally, in the $\pi \pi$ motif the $\mathrm{OH}$ stretching oscillators are symmetry-equivalent and thus fully coupled with a predicted Davydov splitting of about $10 \mathrm{~cm}^{-1}$; the higher-wavenumber anti-symmetric and the lower-wavenumber symmetric combinations are (almost) exclusively IR or Raman active, respectively. 


\subsection{Vibrational Spectra of Propargyl Alcohol Dimers}

The global minimum isomer $g g$-hom- $\mathrm{O}^{g} \pi$ was identified with rotational spectroscopy in a helium expansion by Mani and Arunan[96]. Saini and Viswanathan assigned in addition also the second most stable isomer $g g$-hom- $\mathrm{O}^{t} \pi$ with FTIR spectroscopy in a nitrogen matrix[97]. However, strong overlap between the broad $\mathrm{OH}$ stretching signals of the isomers, and also with a signal of the mixed propargyl alcohol-water dimer, required a complex spectral deconvolution which left room for ambiguity. In comparison, the signals observed here in jet expansions are narrower and thus better resolved. The absence of the matrix environment also makes the comparison with theoretical predictions more straightforward. In Figure 13 the experimental spectra (inner traces) are compared with simulations (outer traces). In agreement with the number concluded from the deconvolution of the matrix spectrum, four bands attributable to dimers are observed at 3581, 3562,3532 and $3507 \mathrm{~cm}^{-1}$. Assuming that the spectral sequence is preserved, matrix shifts relative to the gas phase amount to between -75 and $-92 \mathrm{~cm}^{-1}$. The intensity sequence as well as its change upon switch from FTIR to Raman detection are in very good agreement with the simulation, so that the two most stable isomers can be assigned with confidence. Downshifts relative to the monomer are overestimated by harmonic B3LYP for all four signals, the stronger the higher the $\mathrm{OH} \cdots \mathrm{O}$ character of the fundamental. No signals exclusive to the FTIR or Raman spectrum are observed, indicating negligible population of $\pi \pi$ isomers. This is unlike to dimers of benzyl alcohol, but consistent with their predicted higher relative energies for propargyl alcohol at this level of theory[15]. More strongly downshifted in the spectral region around $3450 \mathrm{~cm}^{-1}$, another band formation is observed clearly in the FTIR and weakly in the Raman spectrum, which scales more steeply with the propargyl alcohol concentration than the dimer signals (Figure 40) and can therefore be attributed to larger clusters, probably trimers. In conclusion, the predicted and experimental spectra for propargyl alcohol dimers are in good agreement with each other and substantiate that no relevant population of higher-energetic isomers with dangling hydroxy groups is expected to perturb the monomer region.

\subsection{Vibrational Spectra of $(+)-\alpha-$-Fenchol Dimers}

In the FTIR and Raman jet spectra of protiated $(+)-\alpha$-fenchol downshifted bands are observed as well, with the most prominent one at $3499 \mathrm{~cm}^{-1}$ (Figure 14). This signal is not present in the FTIR spectrum of the vapor at ambient temperature and gains relative intensity with a higher expansion pressure and a detection farther from the nozzle. It is assigned to the $\mathrm{OH}$ stretch of the donor hydroxy group in the main isomer of the dimer of $(+)$ - $\alpha$-fenchol. Additional weak signals in the same spectral region indicate further minor isomers. The $3499 \mathrm{~cm}^{-1}$ donor signal notably broadens and upshifts when probed very close to the nozzle, which also applies to a lesser extent to the acceptor signal at $3632 \mathrm{~cm}^{-1}$. This might be caused by thermal excitation of low-wavenumber intermolecular vibrations which weakens the hydrogen bond.

In Figure 14 the Raman jet spectra of protiated and deuterated $(+)$ - $\alpha$-fenchol are compared by aligning the wavenumber axes according to $0.73778 \cdot \tilde{v}(\mathrm{OH})=\tilde{v}(\mathrm{OD})$, as suggested by the model of Figure 7 . This highlights the close resemblance in the monomer and dimer acceptor spectral region, barring the different tunneling splittings and impurities. For the deuterated dimer signals are observed at 2680 and $2585 \mathrm{~cm}^{-1}$ for the acceptor and donor, respectively. The position of the latter is underestimated by $4 \mathrm{~cm}^{-1}$ by extrapolation of the model for a fixed fundamental ratio for dangling hydroxy groups. About half of this discrepancy might be explained by a substantially lower harmonic wavenumber, which impacts the isotope effect according to Equation 1. An increase of diagonal anharmonicity upon hydrogen-bonding, as concluded from overtone data for other alcohol dimers[103], might contribute. 


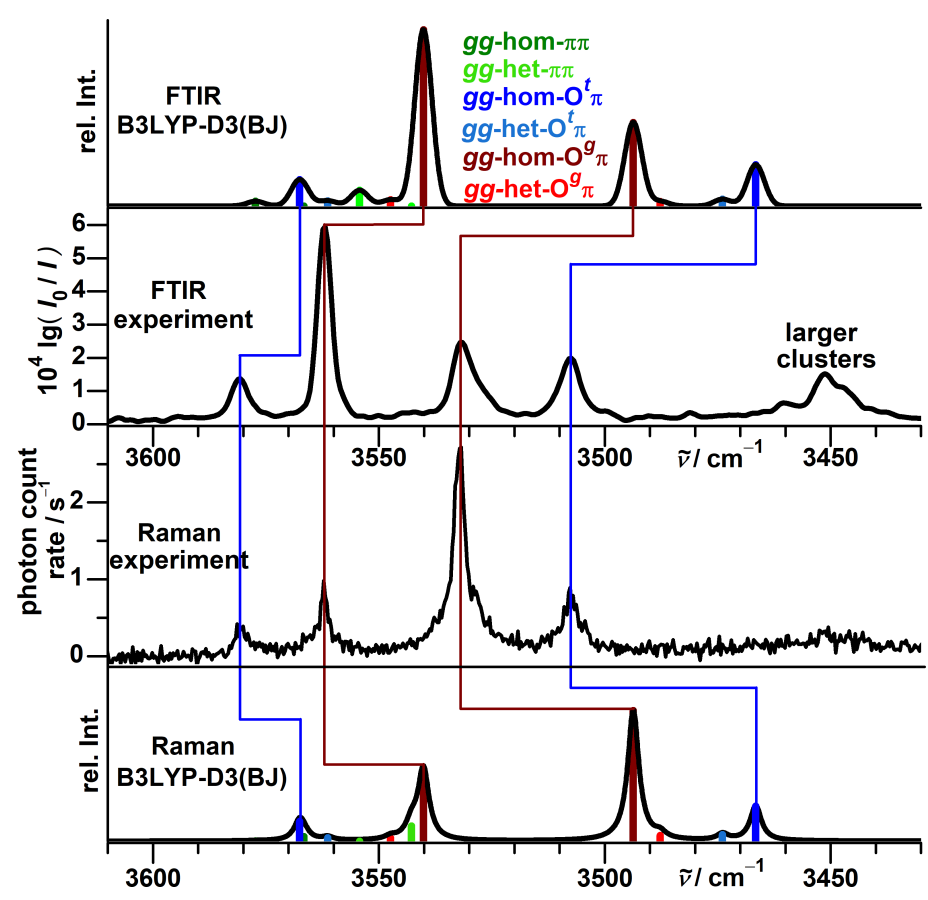

Figure 13. Comparison between experimental (inner) and simulated (outer) FTIR and Raman jet spectra for propargyl alcohol dimers. The simulations assume a conformational temperature of $100 \mathrm{~K}$ and identical vibrational and rotational partition functions except for the rotational symmetry number. In addition, the achirality (non-degeneracy) of $g g$-het- $\pi \pi$ is considered in the statistical weight. Empirically applied are a Gaussian FWHM of $4.5 \mathrm{~cm}^{-1}$ for the FTIR simulation and a Lorentzian FWHM of $3 \mathrm{~cm}^{-1}$ for the Raman simulation. Harmonic $\mathrm{OH}$ stretching wavenumbers are shifted by $-156 \mathrm{~cm}^{-1}$ as needed to match the $g$ monomer spectral center of gravity.

\subsection{Computational Results for $(+)-\alpha$-Fenchol Dimers}

For a hydrogen-bonded dimer of $(+)$ - $\alpha$-fenchol three main conformational degrees of freedom need to to be considered. First, both the donor and acceptor can adopt any of the three intramolecular conformations $g-, g+$ or $t$, resulting in nine combinations. Second, either of the two diastereomeric oxygen electron lone pairs can be coordinated. Third, multiple isomers may exist regarding the torsion about the hydrogen bond, as it can be described by the $\mathrm{C}_{\alpha} \mathrm{OO}^{\prime} \mathrm{C}_{\alpha}^{\prime}$ dihedral. Systematic exploration of this conformational space at the B3LYP-D3(BJ)/def2-TZVP level led to 51 isomers spanning a range of $11 \mathrm{~kJ} \mathrm{~mol}^{-1}$. The six most stable up to $3.1 \mathrm{~kJ} \mathrm{~mol}^{-1}$ were reoptimized with the larger basis set may-cc-pVTZ at the B3LYP-D3(BJ) and PBE0-D3(BJ) levels, listed in Table S13.

The structure of the most stable one, with an advantage of 1.5 (B3LYP) or $1.4 \mathrm{~kJ} \mathrm{~mol}^{-1}$ (PBE0) over the second, is shown in Figure 15. It is constituted of a $g$ - conformer as the donor and a $t$ conformer as the acceptor for the hydrogen bond. The preference for the $g-$ conformer in the donor role was observed before for mixed dimers of $(+)-\alpha-$ fenchol with $(+)$ - and $(-)$ - $\alpha$-pinene[69] as well as the switch to the $t$ conformer when in the acceptor role in the complex of $\alpha$-fenchol with water[104]. The harmonic OH/OD stretching wavenumber of the dangling hydroxy group is predicted to be downshifted by $4 / 3$ (B3LYP) or $7 / 5 \mathrm{~cm}^{-1}$ (PBE0) from the isolated $t$ conformer, which is in qualitative agreement with the observed downshifts for the fundamental transitions of $12 / 9 \mathrm{~cm}^{-1}$. The donor and acceptor modes are predicted to be decoupled enough that OD stretching signals from single- and double-deuterated dimers are expected to coincide within our spectral resolution. The observation of the dimer acceptor band in the Raman but not in the FTIR jet spectrum within the achieved signal-to-noise ratio is in line with its Raman activity being predicted about 4 times but its IR activity about 25 times smaller than for the donor. 


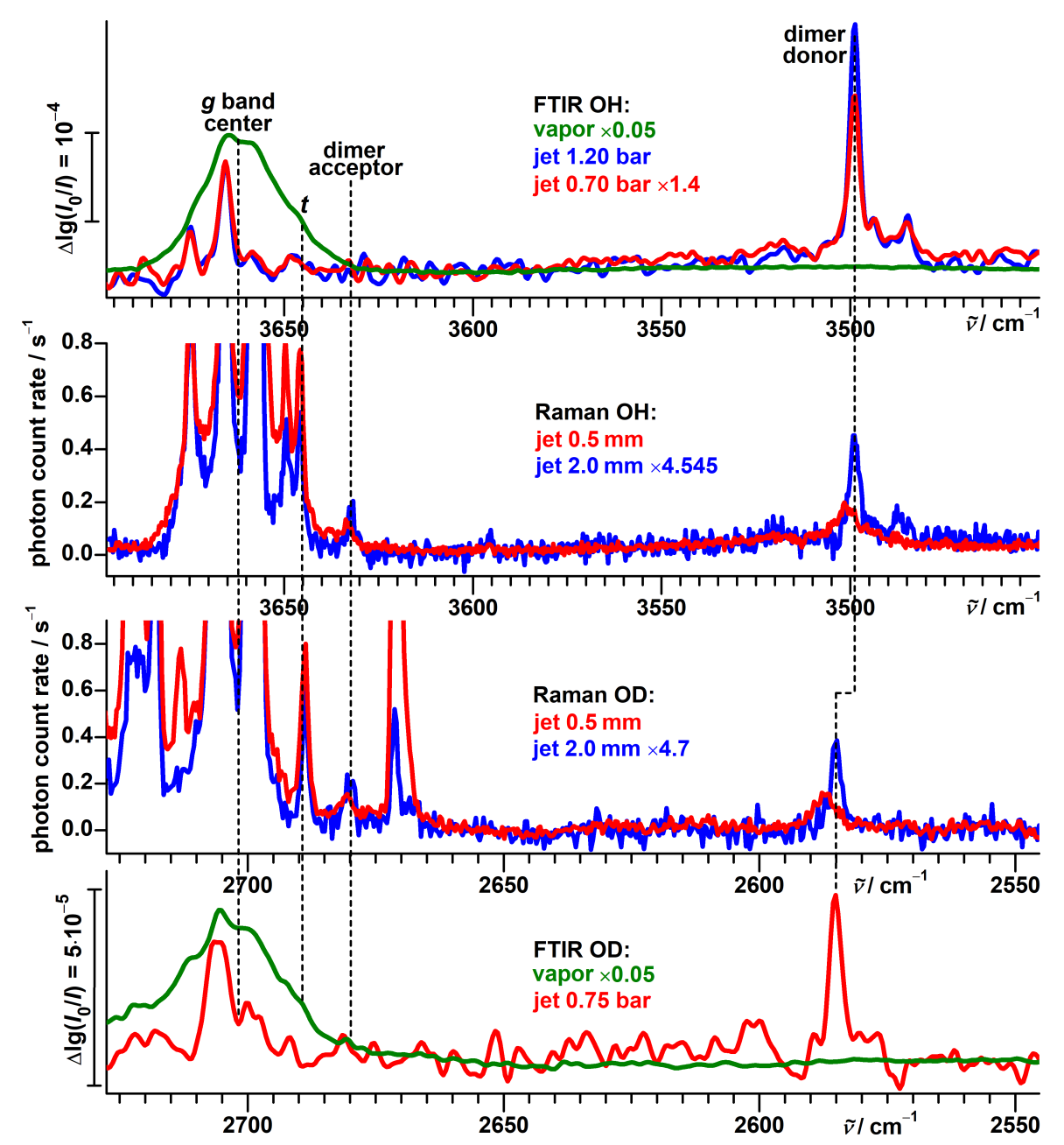

Figure 14. Comparison between FTIR and Raman spectra of protiated (top traces) and deuterated (bottom traces) $(+)$ - $\alpha$-fenchol. The wavenumber axes for the $\mathrm{OH}$ and $\mathrm{OD}$ stretching regions are aligned according to $0.73778 \cdot \tilde{v}(\mathrm{OH})=\tilde{v}(\mathrm{OD})$.

The assignment to this structure is plausible but not rigorous due to calculated $\mathrm{OH}$ stretching properties for dimers being less reliable than for monomers, so that microwave confirmation would be welcome. This might also be interesting in the light of one particular detail: almost $C_{2}$-symmetry is obtained if one disregards the two hydroxy hydrogen atoms (Figure 15). We speculate that these two atoms might be yet again delocalized in this heavy-atom frame, this time within a geared internal rotation which interconverts donor and acceptor, similar to intermolecularly hydrogen-bonded dimers of hydrogen halides[22, 35,105] and intramolecularly hydrogen-bonded diols[106-109], potentially detectable as a splitting with high-resolution spectroscopy.

\section{Conclusions and Outlook}

In this article we inquired the hypothesis of tunneling between two accidentally nearresonant torsional conformers of the monoterpenol $\alpha$-fenchol to explain its anomalous FTIR and Raman jet spectra in the $\mathrm{OH}$ and $\mathrm{OD}$ stretching region. The facilitation of relaxation in jet expansions by tunneling helps to understand the non-observation of transitions for the second conformer in previous microwave jet investigations, despite the calculated very low energy difference in the order of $0.1 \mathrm{~kJ} \mathrm{~mol}^{-1}[46,47]$. By replacing neon with helium as the carrier gas at a lower pressure and detection close to the nozzle, we were able to observe vibrational transitions originating from the elusive second conformer. However, the two lowest conformers might be better classified as torsional states strongly delocalized across both potential wells according to quantum-chemical modelling. This 

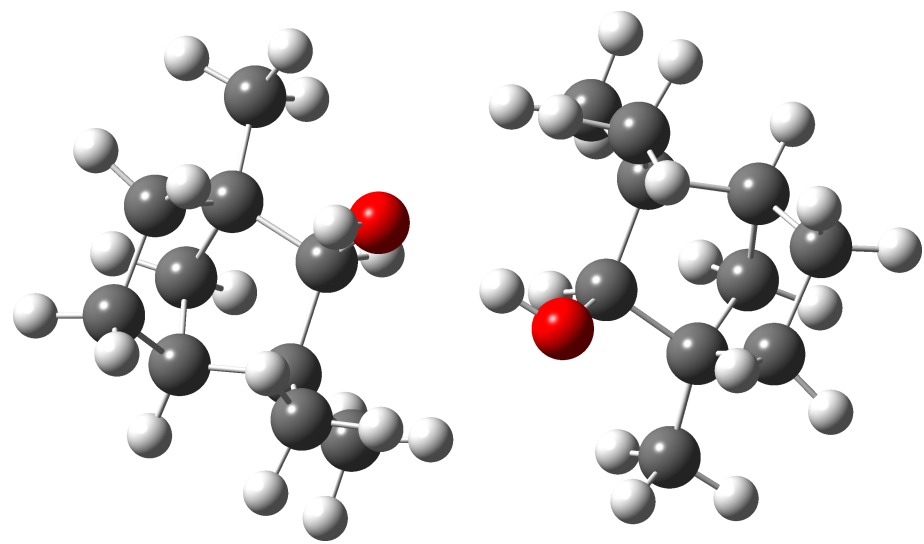

Figure 15. Most stable dimer of $(+)-\alpha$-fenchol at B3LYP/may-cc-pVTZ level viewed along the approximate $C_{2}$ symmetry axis.

partial delocalization is expected to transfer spectral activity to additional transitions, compared to both more strongly asymmetric and fully symmetric species, as validated with the examples of borneol, isopinocampheol and propargyl alcohol.

Observed spectral splittings could be translated into torsional splittings, determined for the main isotopolog of $\alpha$-fenchol as $16(1) \mathrm{cm}^{-1}$ and $9(1) \mathrm{cm}^{-1}$ in the $\mathrm{OH}$ stretch ground and excited state, respectively. As the tunneling interaction largely contributes to these splittings, they are characteristically[85] reduced to 7(3) and 3(1) $\mathrm{cm}^{-1}$ upon increasing the tunneling mass by hydroxy deuteration. Despite being calculated to be less delocalized, the deuterated alcohol shows a more even distribution of Raman activity across the split bands. Based on calculated torsional Franck-Condon factors, this might be explained by a switch of the conformational preference upon OD stretch excitation.

Due to known shortcomings of the Franck-Condon model[93-95] and the sensitivity on the details of the potential, the latter hypothesis however requires further investigation, especially explicit theoretical modelling of relative IR and Raman intensities. Further experimental insights might come from microwave characterization of the second and perhaps also the third torsional state populated in a milder jet expansion or at ambient temperature, a direct measurement of the mm-wave or $\mathrm{THz}$ torsional transitions expected at $16(1)(\mathrm{OH})$ and $7(1) \mathrm{cm}^{-1}(\mathrm{OD})$, as well as analysis of $\mathrm{OH}$ and $\mathrm{OD}$ stretching overtone spectra.

Modification of the carbon skeleton, converting $\alpha$-fenchol into borneol or isopinocampheol, was found to lead to localization and simpler spectra. The rather large molecular structure of $\alpha$-fenchol offers a multitude of additional positions for chemical or isotopic modification to fine-tune the torsional splittings and hydrogen (de-)localization, challenging theory for the calculation of conformational/torsional differences on a $1 \mathrm{~cm}^{-1} h c(\widehat{\approx}$ $0.01 \mathrm{~kJ} \mathrm{~mol}^{-1}$ ) scale. For propargyl alcohol the deuteration of one of the two methylene hydrogen atoms is calculated to introduce an isotopic zero-point asymmetry smaller than the tunneling splitting of the symmetric main isotopolog. We therefore expect this isotopolog to feature a complex $\mathrm{OH}$ stretching Raman jet spectrum similar to the one of $\alpha$-fenchol.

In conclusion, the presented analysis highlights the importance of considering nuclear quantum effects for comprehension of the structure and dynamics of matter, even for cases cases such as asymmetric biomolecules with their (seemingly) well-defined geometries.

Supplementary Materials: Calculations and in part spectra for borneol, isopinocampheol and $\beta$ fenchol; used band positions for the model on the isotope effect; bond length deflection factor derived from the hydroxy radical; benchmarking of the 1D torsion approach with experimental tunneling splittings; details on torsional modelling of $\alpha$-fenchol including dependence of key properties on the asymmetry; list of isomers for dimers of $\alpha$-fenchol and propargyl alcohol; used keywords for calculations; lists of assigned band positions. 
Author Contributions: Conceptualization, R.M., M.A.S.; methodology, R.M., J.R.S.; software, D.L.C, J.R.S.; validation, R.M., J.R.S.; formal analysis, R.M., J.R.S; investigation, R.M., J.R.S.; resources, M.A.S. ; data curation, R.M. ; writing—original draft preparation, R.M., J.R.S.; writing—review and editing, R.M., J.R.S., M.A.S.; visualization, R.M., J.R.S; supervision, M.A.S.; project administration, R.M. ; funding acquisition, M.A.S.. All authors have read and agreed to the published version of the manuscript.

Funding: This project was partly funded by the Deutsche Forschungsgemeinschaft (DFG, German Research Foundation) - 271107160/SPP1807. Computational resources from the GWDG and the Faculty of Chemistry (DFG - 405832858/INST 186/1294-1 FUGG) are acknowledged. The benchmarking aspect has profited from the environment provided by the local research training group BENCh (DFG - 389479699/GRK2455), including a Mercator fellowship for D.L.C. and a junior BENCh grant for J.R.S.

Data Availability Statement: Relevant data is contained within the article and the Supporting Information. In addition, all spectra and leading dimer structures are provided in digital format (.dpt, .xyz) at https://doi.org/10.25625/WMUYHZ [to be published - for review purposes, the unpublished data set may be found under https:/ / data.goettingen-research-online.de/privateurl. xhtml?token=09904e2a-174f-4c7d-a3e1-34ad8c4ae83c]. The 1D torsion code is available on github, at https:/ / github.com/dlc62/Torsion1D. Spectra of $\alpha$-fenchol-OH and the tunneling hypothesis were discussed before in German language as part of the doctoral thesis of R. Medel.[110] Supporting spectra of the deuterated compound and explicit torsional modelling are presented here for the first time.

Acknowledgments: We thank H. Preiß for help with measuring the FTIR spectra for and doing calculations on $\alpha$-fenchol in the context of her undergraduate research participation. We thank A. Nejad for discussions.

Conflicts of Interest: The authors declare no conflict of interest. The funders had no role in the design of the study; in the collection, analyses, or interpretation of data; in the writing of the manuscript, or in the decision to publish the results.

\begin{tabular}{|c|c|}
\hline Abbreviation & \\
\hline e following & breviations are used in this manuscript: \\
\hline ESI & electronic supplementary information \\
\hline FTIR & fourier-transform infrared \\
\hline$g$ & gauche \\
\hline$t$ & trans \\
\hline B3LYP & Becke $\underline{3}$-parameter Lee- $\underline{\text { Yang-Parr hybrid density functional }}$ \\
\hline PBE0 & Perdew-Burke-Ernzerhof $\underline{0}$-parameter hybrid density functional \\
\hline B2PLYP & $\begin{array}{l}\text { Becke 2-parameter 2nd order perturbative Lee-Yang-Parr double-hybrid } \\
\text { density functional }\end{array}$ \\
\hline D3 & $\begin{array}{l}\text { Grimme parametrization of density functional dispersion correction } \\
\text { of } \underline{3} \text { rd generation with two-body terms }\end{array}$ \\
\hline BJ & Becke and Johnson damping function for small interatomic distances \\
\hline MP2 & Møller-Plesset perturbation theory of the 2 nd order \\
\hline DLPNO & domain based local pair-natural orbital methodology \\
\hline $\operatorname{CCSD}(\mathrm{T})$ & coupled-cluster with $\underline{\text { single, }}$ double and perturbative triple excitations \\
\hline $6-311++G(d, p)$ & $\begin{array}{l}\text { Pople basis set with } \underline{6} \text { primitive Gaussian functions for core orbitals and } \\
\text { triple-zeta split } \underline{3}+\underline{1}+\underline{1} \text { primitive Gaussian functions for valence orbitals } \\
\text { with diffuse and polarization functions on heavy and hydrogen atoms }\end{array}$ \\
\hline may-cc-pVTZ & $\begin{array}{l}\text { minimally augmented correlation-consistent polarized } \\
\text { valence-only triple-zeta basis set }\end{array}$ \\
\hline aug-cc-pVQZ & $\begin{array}{l}\text { fully augmented correlation-consistent polarized } \\
\text { valence-only quadruple-zeta basis set }\end{array}$ \\
\hline FCF & Franck-Condon factor \\
\hline
\end{tabular}




\section{References}

1. Schreiner, P.R. Quantum Mechanical Tunneling Is Essential to Understanding Chemical Reactivity. Trends Chem. 2020, 2, 980-989. doi:10.1016/j.trechm.2020.08.006.

2. Quack, M.; Seyfang, G. Atomic and Molecular Tunneling Processes in Chemistry. In Molecular Spectroscopy and Quantum Dynamics; Elsevier, 2021; pp. 231-282. doi:10.1016/B978-0-12-817234-6.00012-X.

3. Rossetti, R.; Rayford, R.; Haddon, R.C.; Brus, L.E. Proton localization in an asymmetric double-minimum potential: 2-methyl-9hydroxyphenalenone. J. Am. Chem. Soc. 1981, 103, 4303-4307. doi:10.1021/ja00405a004.

4. Baughcum, S.L.; Duerst, R.W.; Rowe, W.F.; Smith, Z.; Wilson, E.B. Microwave spectroscopic study of malonaldehyde (3-hydroxy2-propenal). 2. Structure, dipole moment, and tunneling. J. Am. Chem. Soc. 1981, 103, 6296-6303. doi:10.1021/ja00411a005.

5. Redington, R.L.; Redington, T.E.; Hunter, M.A.; Field, R.W. $\tilde{A}^{1} B_{2}-\tilde{X}^{1} A_{1} 26^{v}{ }_{0}$ transitions of ${ }^{18}$ O-enriched tropolone. J. Chem. Phys. 1990, 92, 6456-6462. doi:10.1063/1.458325.

6. Busch, J.H.; De la Vega, J.R. Symmetry and tunneling in proton transfer reactions. Proton exchange between methyloxonium ion and methyl alcohol, methyl alcohol and methoxide ion, hydronium ion and water, and water and hydroxyl ion. J. Am. Chem. Soc. 1977, 99, 2397-2406. doi:10.1021/ja00450a001.

7. Bosch, E.; Moreno, M.; Lluch, J.M. Tunneling dynamics in isotopically substituted malonaldehyde. Comparison between symmetric and asymmetric species. J. Am. Chem. Soc. 1992, 114, 2072-2076. doi:10.1021/ja00032a021.

8. Cordes, J.; Das, A. Tunnelling in asymmetric double-well potentials: varying initial states. Superlattices Microstruct. 2001, 29, 121-132. doi:10.1006/spmi.2000.0964.

9. Song, D.Y. Tunneling and energy splitting in an asymmetric double-well potential. Ann. Phys. 2008, 323, 2991-2999. doi:10.1016/j.aop.2008.09.004.

10. Rastelli, G. Semiclassical formula for quantum tunneling in asymmetric double-well potentials. Phys. Rev. A 2012, 86, 012106. doi:10.1103/PhysRevA.86.012106.

11. Song, D.Y. Localization or tunneling in asymmetric double-well potentials. Ann. Phys. 2015, 362, 609-620. doi:10.1016/j.aop.2015.08.029.

12. Bredtmann, T.; Manz, J.; Zhao, J.M. Concerted Electronic and Nuclear Fluxes During Coherent Tunnelling in Asymmetric Double-Well Potentials. J. Phys. Chem. A 2016, 120, 3142-3154. doi:10.1021/acs.jpca.5b11295.

13. Halataei, S.M.H.; Leggett, A.J. Tunnel Splitting in Asymmetric Double Well Potentials: An Improved WKB Calculation. ArXiv170305758v3 Quant-Ph 2017, [arXiv:quant-ph/1703.05758v3].

14. Jahr, E.; Laude, G.; Richardson, J.O. Instanton theory of tunneling in molecules with asymmetric isotopic substitutions. J. Chem. Phys. 2020, 153, 094101. doi:10.1063/5.0021831.

15. Medel, R.; Suhm, M.A. Understanding benzyl alcohol aggregation by chiral modification: the pairing step. Phys. Chem. Chem. Phys. 2020, 22, 25538-25551. doi:10.1039/D0CP04825A.

16. De la Vega, J.R. Role of symmetry in the tunneling of the proton in double-minimum potentials. Acc. Chem. Res. 1982, 15, 185-191. doi:10.1021/ar00078a004.

17. Pejlovas, A.M.; Lin, W.; Kukolich, S.G. Microwave spectra and structure of the cyclopropanecarboxylic acid-formic acid dimer. J. Chem. Phys. 2015, 143, 124311. doi:10.1063/1.4931923.

18. Evangelisti, L.; Caminati, W. Modeling the internal rotation tunnelling in benzyl alcohol by ring fluorination: The rotational spectrum of 3,5-difluorobenzyl alcohol. Chem. Phys. Lett. X 2019, 1, 100004. doi:10.1016/j.cpletx.2018.100004.

19. Thomas, J.; Carrillo, M.J.; Serrato, A.; Xie, F.; Jäger, W.; Xu, Y.; Lin, W. Microwave spectrum of the complex of 3,3,3-trifluoro-2(trifluoromethyl)propanoic acid and formic acid. Mol. Phys. 2019, 117, 1193-1199. doi:10.1080/00268976.2018.1532539.

20. Kakar, R.K.; Quade, C.R. Microwave rotational spectrum and internal rotation in gauche ethyl alcohol. J. Chem. Phys. 1980, 72, 4300-4307. doi:10.1063/1.439723.

21. Sekiya, H.; Takesue, H.; Nishimura, Y.; Li, Z.H.; Mori, A.; Takeshita, H. Electronic spectra of jet-cooled isopropyltropolones in the $S_{1}-S_{0}$ region. Proton tunneling in the $S_{1}$ state. J. Chem. Phys. 1990, 92, 2790-2796. doi:10.1063/1.457925.

22. Farrell, J.T.; Suhm, M.A.; Nesbitt, D.J. Breaking symmetry with hydrogen bonds: Vibrational predissociation and isomerization dynamics in HF-DF and DF-HF isotopomers. J. Chem. Phys. 1996, 104, 9313-9331. doi:10.1063/1.471677.

23. Su, C.F.; Quade, C. Microwave Spectra of gauche $\mathrm{CH}_{2} \mathrm{DCH}_{2} \mathrm{OH}$ Including Excited States of the -OH Torsion. J. Mol. Spectrosc. 1998, 188, 1-8. doi:10.1006/jmsp.1997.7478.

24. Lesarri, A.; Shipman, S.T.; Neill, J.L.; Brown, G.G.; Suenram, R.D.; Kang, L.; Caminati, W.; Pate, B.H. Interplay of Phenol and Isopropyl Isomerism in Propofol from Broadband Chirped-Pulse Microwave Spectroscopy. J. Am. Chem. Soc. 2010, 132, 13417-13424. doi:10.1021/ja104950w.

25. Albert, S.; Lerch, P.; Prentner, R.; Quack, M. Tunneling and Tunneling Switching Dynamics in Phenol and Its Isotopomers from High-Resolution FTIR Spectroscopy with Synchrotron Radiation. Angew. Chem. Int. Ed. 2013, 52, 346-349. doi:10.1002/anie.201205990.

26. Albert, S.; Chen, Z.; Fábri, C.; Lerch, P.; Prentner, R.; Quack, M. A combined Gigahertz and Terahertz (FTIR) spectroscopic investigation of meta-D-phenol: observation of tunnelling switching. Mol. Phys. 2016, 114, 2751-2768. doi:10.1080/00268976.2016.1226444.

27. Saragi, R.T.; Juanes, M.; Caminati, W.; Lesarri, A.; Enríquez, L.; Jaraíz, M. Rotational Spectrum, Tunneling Motions, and Intramolecular Potential Barriers in Benzyl Mercaptan. J. Phys. Chem. A 2019, 123, 8435-8440. doi:10.1021/acs.jpca.9b06921. 
28. Li, W.; Tikhonov, D.; Schnell, M. Double proton transfer across a table: the formic acid dimer - fluorobenzene complex. Angew. Chem. 2021, p. ange.202108242. doi:10.1002/ange.202108242.

29. Ensminger, F.A.; Plassard, J.; Zwier, T.S.; Hardinger, S. Vibronically state-selective photoisomerization in 5-hydroxytropolone. J. Chem. Phys. 1993, 99, 8341-8344. doi:10.1063/1.465607.

30. Barclay, A.J.; Pietropolli Charmet, A.; Michaelian, K.H.; McKellar, A.R.W.; Moazzen-Ahmadi, N. Micro-solvation of CO in water: infrared spectra and structural calculations for $\left(\mathrm{D}_{2} \mathrm{O}\right)_{2}-\mathrm{CO}$ and $\left(\mathrm{D}_{2} \mathrm{O}\right)_{3}-\mathrm{CO}$. Phys. Chem. Chem. Phys. 2019, 21, 26564-26568. doi:10.1039/C9CP05480D.

31. Wassermann, T.N.; Luckhaus, D.; Coussan, S.; Suhm, M.A. Proton tunneling estimates for malonaldehyde vibrations from supersonic jet and matrix quenching experiments. Phys. Chem. Chem. Phys. 2006, 8, 2344-2348. doi:10.1039/b602319n.

32. Lüttschwager, N.O.; Wassermann, T.N.; Coussan, S.; Suhm, M.A. Vibrational tuning of the Hydrogen transfer in malonaldehyde a combined FTIR and Raman jet study. Mol. Phys. 2013, 111, 2211-2227. doi:10.1080/00268976.2013.798042.

33. Rambaud, C.; Trommsdorff, H. Cooperative proton transfer and tunneling in dye doped benzoic acid crystals. Chem. Phys. Lett. 1999, 306, 124-132. doi:10.1016/S0009-2614(99)00447-9.

34. Yamada, K.M.; Ross, S.C. Isomerisation: Don't forget the possibility of enhanced tunnelling! A simple two-state model for the on-resonance and near-resonance behaviour of enhanced tunnelling. J. Mol. Struct. 2006, 795, 84-92. doi:10.1016/j.molstruc.2006.02.021.

35. Schuder, M.D.; Lovejoy, C.M.; Lascola, R.; Nesbitt, D.J. High resolution, jet-cooled infrared spectroscopy of $(\mathrm{HCl})_{2}:$ Analysis of $v_{1}$ and $v_{2} \mathrm{HCl}$ stretching fundamentals, interconversion tunneling, and mode-specific predissociation lifetimes. J. Chem. Phys. 1993, 99, 4346-4362. doi:10.1063/1.466089.

36. Schuder, M.D.; Nelson, D.D.; Nesbitt, D.J. Slit-jet near-infrared diode laser spectroscopy of $(\mathrm{DCl})_{2}: v_{1}, v_{2} \mathrm{DCl}$ stretching fundamentals, tunneling dynamics, and the influence of large amplitude "geared" intermolecular rotation. J. Chem. Phys. 1993, 99, 5045-5060. doi:10.1063/1.466005.

37. Gil, M.; Jasny, J.; Vogel, E.; Waluk, J. Ground and excited state tautomerization in 9-acetoxy-2,7,12,17-tetra-n-propylporphycene. Chem. Phys. Lett. 2000, 323, 534-541. doi:10.1016/S0009-2614(00)00574-1.

38. Vdovin, A.; Sepioł, J.; Urbańska, N.; Pietraszkiewicz, M.; Mordziński, A.; Waluk, J. Evidence for Two Forms, Double Hydrogen Tunneling, and Proximity of Excited States in Bridge-Substituted Porphycenes: Supersonic Jet Studies. J. Am. Chem. Soc. 2006, 128, 2577-2586. doi:10.1021/ja054745m.

39. Fita, P.; Garbacz, P.; Nejbauer, M.; Radzewicz, C.; Waluk, J. Ground and Excited State Double Hydrogen Transfer in Symmetric and Asymmetric Potentials: Comparison of 2,7,12,17-Tetran-propylporphycene with 9-Acetoxy-2,7,12,17-tetran-propylporphycene. Chem. Eur. J. 2011, 17, 3672-3678. doi:10.1002/chem.201002931.

40. Mengesha, E.T.; Zehnacker-Rentien, A.; Sepioł, J.; Kijak, M.; Waluk, J. Spectroscopic Study of Jet-Cooled Deuterated Porphycenes: Unusual Isotopic Effects on Proton Tunneling. J. Phys. Chem. B 2015, 119, 2193-2203. doi:10.1021/jp505553z.

41. Peukert, S.; Kijak, M.; Ostapko, J.; Sepioł, J.; Le Bris, C.; Zehnacker-Rentien, A.; Gil, M.; Waluk, J. Supersonic jet spectroscopy of parent hemiporphycene: Structural assignment and vibrational analysis for $\mathrm{S}_{0}$ and $\mathrm{S}_{1}$ electronic states. J. Chem. Phys. 2018, 149, 134307. doi:10.1063/1.5048843.

42. Bondybey, V.E.; Haddon, R.C.; English, J.H. Fluorescence and phosphorescence of 9-hydroxyphenalenone in solid neon and its hydrogen tunneling potential function. J. Chem. Phys. 1984, 80, 5432-5437. doi:10.1063/1.446650.

43. Kunze, K.L.; De la Vega, J.R. Intramolecular proton exchange in 9-hydroxyphenalen-1-one and methyl-9-hydroxyphenalen-1-one. J. Am. Chem. Soc. 1984, 106, 6528-6533. doi:10.1021/ja00334a012.

44. Nishi, K.; Sekiya, H.; Mochida, T.; Sugawara, T.; Nishimura, Y. Coupling between the internal rotation of the methyl group and proton/deuteron transfer in jet-cooled 5-methyl-9-hydroxyphenalenone(OH) and 5-methyl-9-hydroxyphenalenone(OD): Tunneling rate dependence of coupling potential. J. Chem. Phys. 2000, 112, 5002-5011. doi:10.1063/1.481055.

45. Mori, H.; Sekiya, H.; Miyoshi, E.; Mogi, K.; Sakai, Y. Effects of intermolecular interaction on proton tunneling: Theoretical study on two-dimensional potential energy surfaces for 9-hydroxyphenalenone- $\mathrm{CO}_{2} / \mathrm{H}_{2} \mathrm{O}$ complexes. J. Chem. Phys. 2003, 119, 4159-4165. doi:10.1063/1.1592505.

46. Pate, B.H.; Evangelisti, L.; Caminati, W.; Xu, Y.; Thomas, J.; Patterson, D.; Perez, C.; Schnell, M. Quantitative Chiral Analysis by Molecular Rotational Spectroscopy. In Chiral Analysis; Elsevier, 2018; pp. 679-729. doi:10.1016/B978-0-444-64027-7.00019-7.

47. Neeman, E.M.; Huet, T.R. Identification of the maze in the conformational landscape of fenchol. Phys. Chem. Chem. Phys. 2018, 20, 24708-24715. doi:10.1039/C8CP04011G.

48. Medel, R.; Suhm, M.A. Predicting OH stretching fundamental wavenumbers of alcohols for conformational assignment: different correction patterns for density functional and wave-function-based methods. Phys. Chem. Chem. Phys. 2021, 23, 5629-5643. doi:10.1039/D1CP00342A.

49. Forsting, T.; Suhm, M. Curry-Jet SETUP. doi:10.6084/m9.figshare.6395840.v1.

50. Suhm, M.A.; Kollipost, F. Femtisecond single-mole infrared spectroscopy of molecular clusters. Phys. Chem. Chem. Phys. 2013, 15, 10702. doi:10.1039/c3cp51515j.

51. Frisch, M.J.; Trucks, G.W.; Schlegel, H.B.; Scuseria, G.E.; Robb, M.A.; Cheeseman, J.R.; Scalmani, G.; Barone, V.; Mennucci, B.; Petersson, G.A.; Nakatsuji, H.; Caricato, M.; Li, X.; Hratchian, H.P.; Izmaylov, A.F.; Bloino, J.; Zheng, G.; Sonnenberg, J.L.; Hada, M.; Ehara, M.; Toyota, K.; Fukuda, K.; Hasegawa, J.; Ishida, M.; Nakajima, T.; Honda, Y.; Kitao, O.; Nakai, H.; Vreven, T.; Montgomery, J.A.J.; Peralta, J.E.; Ogliaro, F.; Bearpark, M.; Heyd, J.J.; Brothers, E.; Kudin, K.N.; Staroverov, V.N.; Kobayashi, R.; 
Normand, J.; Raghavachari, K.; Rendell, A.; Burant, J.C.; Iyengar, S.S.; Tomasi, J.; Cossi, M.; Rega, N.; Millam, J.M.; Klene, M.; Knox, J.E.; Cross, J.B.; Bakken, V.; Adamo, C.; Jaramillo, J.; Gomperts, R.; Stratmann, R.E.; Yazyev, O.; Austin, A.J.; Cammi, R.; Pomelli, C.; Ochterski, J.W.; Martin, R.L.; Morokuma, K.; Zakrzewski, V.G.; Voth, G.A.; Salvador, P.; Dannenberg, J.J.; Dapprich, S.; Daniels, A.D.; Farkas, Ö.; Foresman, J.B.; Ortiz, J.V.; Cioslowski, J.; Fox, D.J. Gaussian 09, Revision E.01. Technical report, Gaussian, Inc., Wallingford CT, 2009.

52. Becke, A.D. Density-functional exchange-energy approximation with correct asymptotic behavior. Phys. Rev. A: At., Mol., Opt. Phys. 1988, 38, 3098-3100. doi:10.1103/PhysRevA.38.3098.

53. Lee, C.; Yang, W.; Parr, R.G. Development of the Colle-Salvetti correlation-energy formula into a functional of the electron density. Phys. Rev. B: Condens. Matter Mater. Phys. 1988, 37, 785-789. doi:10.1103/PhysRevB.37.785.

54. Becke, A.D. Density-functional thermochemistry. III. The role of exact exchange. J. Chem. Phys. 1993, 98, 5648-5652. doi:10.1063/1.464913.

55. Stephens, P.J.; Devlin, F.J.; Chabalowski, C.F.; Frisch, M.J. Ab Initio Calculation of Vibrational Absorption and Circular Dichroism Spectra Using Density Functional Force Fields. J. Phys. Chem. 1994, 98, 11623-11627. doi:10.1021/j100096a001.

56. Adamo, C.; Barone, V. Toward reliable density functional methods without adjustable parameters: The PBE0 model. J. Chem. Phys. 1999, 110, 6158-6170. doi:10.1063/1.478522.

57. Grimme, S. Semiempirical hybrid density functional with perturbative second-order correlation. J. Chem. Phys. 2006, 124, 034108. doi:10.1063/1.2148954.

58. Grimme, S.; Ehrlich, S.; Goerigk, L. Effect of the damping function in dispersion corrected density functional theory. J. Comput. Chem. 2011, 32, 1456-1465. doi:10.1002/jcc.21759.

59. Papajak, E.; Zheng, J.; Xu, X.; Leverentz, H.R.; Truhlar, D.G. Perspectives on Basis Sets Beautiful: Seasonal Plantings of Diffuse Basis Functions. J. Chem. Theory Comput. 2011, 7, 3027-3034. doi:10.1021/ct200106a.

60. Riplinger, C.; Neese, F. An efficient and near linear scaling pair natural orbital based local coupled cluster method. J. Chem. Phys. 2013, 138, 034106. doi:10.1063/1.4773581.

61. Riplinger, C.; Sandhoefer, B.; Hansen, A.; Neese, F. Natural triple excitations in local coupled cluster calculations with pair natural orbitals. J. Chem. Phys. 2013, 139, 134101. doi:10.1063/1.4821834.

62. Riplinger, C.; Pinski, P.; Becker, U.; Valeev, E.F.; Neese, F. Sparse maps-A systematic infrastructure for reduced-scaling electronic structure methods. II. Linear scaling domain based pair natural orbital coupled cluster theory. J. Chem. Phys. 2016, 144, 024109. doi:10.1063/1.4939030.

63. Neese, F. Software update: the ORCA program system, version 4.0. WIREs Comput Mol Sci 2018, 8. doi:10.1002/wcms.1327.

64. Oswald, S. Weak Hydrogen Bonds to Molecular Nitrogen and Oxygen as Experimental Benchmarks for Quantum Chemistry; Dissertation: Georg-August-Universität Göttingen, 2019.

65. Pearson, J.; Sastry, K.; Herbst, E.; De Lucia, F.C. The Millimeter- and Submillimeter-Wave Spectrum of Gauche-Ethyl Alcohol. J. Mol. Spectrosc. 1996, 175, 246-261. doi:10.1006/jmsp.1996.0030.

66. Chung-Phillips, A. Torsional energy levels and wave functions. J. Comput. Chem. 1992, 13, 874-882. doi:10.1002/jcc.540130712.

67. Dieke, G.; Crosswhite, H. The ultraviolet bands of OH Fundamental data. J. Quant. Spectrosc. Radiat. Transf. 1962, 2, 97-199. doi:10.1016/0022-4073(62)90061-4.

68. Coxon, J.A. The $\mathrm{A}^{2} \Sigma^{+}-\mathrm{X}^{2} \Pi_{i}$ system of OD. J. Mol. Spectrosc. 1975, 58, 1-28. doi:10.1016/0022-2852(75)90153-8.

69. Medel, R.; Stelbrink, C.; Suhm, M.A. Vibrational Signatures of Chirality Recognition Between $\alpha$-Pinene and Alcohols for Theory Benchmarking. Angew. Chem. Int. Ed. 2019, 58, 8177-8181. doi:10.1002/anie.201901687.

70. Zielke, P. Ramanstreuung am Überschallstrahl: Wasserstoffbrückendynamik aus neuer Perspektive; Dissertation: Georg-AugustUniversität Göttingen, 2007.

71. Wassermann, T.N.; Suhm, M.A. Ethanol Monomers and Dimers Revisited: A Raman Study of Conformational Preferences and Argon Nanocoating Effects. J. Phys. Chem. A 2010, 114, 8223-8233. doi:10.1021/jp104861q.

72. Wassermann, T.N.; Suhm, M.A.; Roubin, P.; Coussan, S. Isomerization around C-C and C-O bonds in 1-propanol: Collisional relaxation in supersonic jets and selective IR photo-isomerization in cryogenic matrices. J. Mol. Struct. 2012, 1025, 20-32. doi:10.1016/j.molstruc.2011.12.034.

73. Avila, G.; Fernández, J.; Tejeda, G.; Montero, S. The Raman spectra and cross-sections of $\mathrm{H}_{2} \mathrm{O}, \mathrm{D}_{2} \mathrm{O}$, and $\mathrm{HDO}$ in the OH/OD stretching regions. J. Mol. Spectrosc. 2004, 228, 38-65. doi:10.1016/j.jms.2004.06.012.

74. Otto, K.E.; Xue, Z.; Zielke, P.; Suhm, M.A. The Raman spectrum of isolated water clusters. Phys. Chem. Chem. Phys. 2014, 16, 9849. doi:10.1039/c3cp54272f.

75. Larsen, R.W.; Zielke, P.; Suhm, M.A. Hydrogen-bonded OH stretching modes of methanol clusters: A combined IR and Raman isotopomer study. J. Chem. Phys. 2007, 126, 194307. doi:10.1063/1.2732745.

76. Schiel, D.; Richter, W. Effect of the internal rotation of the $\mathrm{OH}$ group on the $\mathrm{OH}$ stretching vibrational Raman profile of ethanol in the gas phase. The Journal of Chemical Physics 1983, 78, 6559-6562. doi:10.1063/1.444703.

77. Schuder, M.D.; Nesbitt, D.J. High resolution near infrared spectroscopy of $\mathrm{HCl}-\mathrm{DCl}$ and DCl-HCl: Relative binding energies, isomer interconversion rates, and mode specific vibrational predissociation. J. Chem. Phys. 1994, 100, $7250-7267$. doi:10.1063/1.466872.

78. Subramanian, R.; Novick, S.E.; Bohn, R.K. Torsional analysis of 2-butynol. J. Mol. Spectrosc. 2003, 222, 57-62. doi:10.1016/S00222852(03)00170-X. 
79. Hirota, E. Internal rotation in propargyl alcohol from microwave spectrum. J. Mol. Spectrosc. 1968, 26, 335-350. doi:10.1016/00222852(68)90069-6.

80. Pearson, J.; Drouin, B. The ground state torsion-rotation spectrum of propargyl alcohol $\left(\mathrm{HCCCH}_{2} \mathrm{OH}\right)$. J. Mol. Spectrosc. 2005, 234, 149-156. doi:10.1016/j.jms.2005.08.013.

81. Mani, D.; Arunan, E. Microwave Spectroscopic and Atoms in Molecules Theoretical Investigations on the Ar ...Propargyl Alcohol Complex: Ar $\cdots H-O$, Ar $\cdots \pi$, and Ar ‥C Interactions. ChemPhysChem 2013, 14, 754-763. doi:10.1002/cphc.201200760.

82. Ruoff, R.S.; Klots, T.D.; Emilsson, T.; Gutowsky, H.S. Relaxation of conformers and isomers in seeded supersonic jets of inert gases. J. Chem. Phys. 1990, 93, 3142-3150. doi:10.1063/1.458848.

83. Herzberg, G. Molecular spectra and molecular structure. Volume 1: Spectra of diatomic molecules, 2. ed.; 23. print ed.; van Nostrand: New York, 1987.

84. Noether, H.D. The Ratio Rule. J. Chem. Phys. 1943, 11, 97-98. doi:10.1063/1.1723809.

85. Medel, R. Simple models for the quick estimation of ground state hydrogen tunneling splittings in alcohols and other compounds. Phys. Chem. Chem. Phys. 2021, 23, 17591-17605. doi:10.1039/D1CP02115J.

86. Hirota, E.; Kawashima, Y. Internal Rotation of the Hydroxyl Group in Isopropanol and the Chirality of the Gauche Form: Fourier Transform Microwave Spectroscopy of $\left(\mathrm{CH}_{3}\right)_{2}$ CHOD. J. Mol. Spectrosc. 2001, 207, 243-253. doi:10.1006/jmsp.2001.8352.

87. Juanes, M.; Li, W.; Spada, L.; Evangelisti, L.; Lesarri, A.; Caminati, W. Internal dynamics of cyclohexanol and the cyclohexanol-water adduct. Phys. Chem. Chem. Phys. 2019, 21, 3676-3682. doi:10.1039/C8CP04455D.

88. Macdonald, J.N.; Norbury, D.; Sheridan, J. Microwave spectrum, dipole moment and internal rotation potential function of gauche-cyclopropanol. J. Chem. Soc. Faraday Trans. 2 1978, 74, 1365. doi:10.1039/f29787401365.

89. Giuliano, B.M.; Caminati, W. Isotopomeric Conformational Change in Anisole-Water. Angew. Chem. Int. Ed. 2005, 44, 603-606. doi:10.1002/anie.200461860.

90. Zhou, J.; Kye, Y.S.; Harbison, G.S. Isotopomeric Polymorphism. J. Am. Chem. Soc. 2004, 126, 8392-8393. doi:10.1021/ja0479843.

91. Sibert, E.L.; Castillo-Chará, J. Theoretical studies of the potential surface and vibrational spectroscopy of $\mathrm{CH}_{3} \mathrm{OH}$ and its deuterated analogs. J. Chem. Phys. 2005, 122, 194306. doi:10.1063/1.1898211.

92. Dübal, H.R.; Crim, F.F. Vibrational overtone predissociation spectroscopy of hydrogen peroxide. J. Chem. Phys. 1985, 83, 3863-3872. doi:10.1063/1.449097.

93. Matthews, J.; Martínez-Avilés, M.; Francisco, J.S.; Sinha, A. Probing OH stretching overtones of $\mathrm{CH}_{3} \mathrm{OOH}$ through action spectroscopy: Influence of dipole moment dependence on HOOC torsion. J. Chem. Phys. 2008, 129, 074316. doi:10.1063/1.2967185.

94. Hansen, A.S.; Huchmala, R.M.; Vogt, E.; Boyer, M.A.; Bhagde, T.; Vansco, M.F.; Jensen, C.V.; Kjærsgaard, A.; Kjaergaard, H.G.; McCoy, A.B.; Lester, M.I. Coupling of torsion and OH-stretching in tert-butyl hydroperoxide. I. The cold and warm first OH-stretching overtone spectrum. J. Chem. Phys. 2021, 154, 164306. doi:10.1063/5.0048020.

95. Vogt, E.; Huchmala, R.M.; Jensen, C.V.; Boyer, M.A.; Wallberg, J.; Hansen, A.S.; Kjærsgaard, A.; Lester, M.I.; McCoy, A.B.; Kjaergaard, H.G. Coupling of torsion and $\mathrm{OH}$-stretching in tert-butyl hydroperoxide. II. The OH-stretching fundamental and overtone spectra. J. Chem. Phys. 2021, 154, 164307. doi:10.1063/5.0048022.

96. Mani, D.; Arunan, E. Rotational spectra of propargyl alcohol dimer: A dimer bound with three different types of hydrogen bonds. J. Chem. Phys. 2014, 141, 164311. doi:10.1063/1.4898378.

97. Saini, J.; Viswanathan, K.S. Discerning Near-Isoergic Isomers. A Matrix Isolation Infrared and ab Initio Study of the Propargyl Alcohol Dimers. J. Phys. Chem. A 2017, 121, 1448-1459. doi:10.1021/acs.jpca.6b12702.

98. Nyquist, R. Vibrational assignments of $\mathrm{H}-\mathrm{CC}-\mathrm{CH}_{2} \mathrm{OH}$ and its deuterium analogs. Spectrochimica Acta Part A: Molecular Spectroscopy 1971, 27, 2513-2523. doi:10.1016/0584-8539(71)80148-4.

99. Hudspeth, E.; McWhorter, D.A.; Pate, B.H. Intramolecular vibrational energy redistribution in the acetylenic C-H and hydroxyl stretches of propynol. J. Chem. Phys. 1998, 109, 4316-4326. doi:10.1063/1.477075.

100. Sundararajan, K.; Gopi, R.; Ramanathan, N. Conformations of propargyl alcohol and its interaction with acetylene: A matrix isolation infrared and DFT computations. J. Mol. Struct. 2016, 1121, 26-34. doi:10.1016/j.molstruc.2016.05.047.

101. Altnöder, J.; Bouchet, A.; Lee, J.J.; Otto, K.E.; Suhm, M.A.; Zehnacker-Rentien, A. Chirality-dependent balance between hydrogen bonding and London dispersion in isolated $( \pm)-1$-indanol clusters. Phys. Chem. Chem. Phys. 2013, $15,10167$. doi:10.1039/c3cp50708d.

102. Altnöder, J.; Oswald, S.; Suhm, M.A. Phenyl- vs Cyclohexyl-Substitution in Methanol: Implications for the OH Conformation and for Dispersion-Affected Aggregation from Vibrational Spectra in Supersonic Jets. J. Phys. Chem. A 2014, 118, 3266-3279. doi:10.1021/jp501763b.

103. Kollipost, F.; Papendorf, K.; Lee, Y.F.; Lee, Y.P.; Suhm, M.A. Alcohol dimers - how much diagonal OH anharmonicity? Phys. Chem. Chem. Phys. 2014, 16, 15948-15956. doi:10.1039/C4CP01418A.

104. Neeman, E.M.; Huet, T.R. Conformational changes in hydroxyl functional groups upon hydration: the case study of endo fenchol. Phys. Chem. Chem. Phys. 2021, 23, 2179-2185. doi:10.1039/D0CP06042A.

105. Castillo-Chará, J.; McIntosh, A.L.; Wang, Z.; Lucchese, R.R.; Bevan, J.W. Near-infrared spectra and rovibrational dynamics on a four-dimensional ab initio potential energy surface of (HBr) 2 . J. Chem. Phys. 2004, 120, 10426-10441. doi:10.1063/1.1736632.

106. Christen, D.; Coudert, L.; Larsson, J.; Cremer, D. The Rotational-Torsional Spectrum of the g'Gg Conformer of Ethylene Glycol: Elucidation of an Unusual Tunneling Path. J. Mol. Spectrosc. 2001, 205, 185-196. doi:10.1006/jmsp.2000.8263. 
107. Paul, J.; Hearn, I.; Howard, B.J. Chiral recognition in a single molecule: a study of homo and heterochiral butan-2,3-diol by Fourier transform microwave spectroscopy. Mol. Phys. 2007, 105, 825-839. doi:10.1080/00268970701241649.

108. Olschewski, M.; Lindner, J.; Vöhringer, P. A Hydrogen-Bond Flip-Flop through a Bjerrum-Type Defect. Angew. Chem. Int. Ed. 2013, 52, 2602-2605. doi:10.1002/anie.201208625.

109. Evangelisti, L.; Gou, Q.; Spada, L.; Feng, G.; Caminati, W. Conformational analysis of 1,4-butanediol: A microwave spectroscopy study. Chem. Phys. Lett. 2013, 556, 55-58. doi:10.1016/j.cplett.2012.11.080.

110. Medel, R. Schwingungsspektroskopische Untersuchungen zur Chiralitätserkennung und Torsionsdynamik bei Alkoholen; PhD dissertation: Georg-August-Universität Göttingen, 2020.

111. Heger, M. Diagonal and Off-Diagonal Anharmonicity in Hydrogen-Bonded Systems; Dissertation: Georg-August-Universität-Göttingen, 2016.

112. Lüttschwager, N.O.B. Raman Spectroscopy of Conformational Rearrangements at Low Temperatures Folding and Stretching of Alkanes in Supersonic Jets; Dissertation, Springer International Publishing: Cham, 2014.

113. Gawrilow, M.; Suhm, M.A. 2-Methoxyethanol: harmonic tricks, anharmonic challenges and chirality-sensitive chain aggregation. Phys. Chem. Chem. Phys. 2020, 22, 15303-15311. doi:10.1039/D0CP02488K.

114. Moruzzi, G.; Xu, L.; Lees, R.; Winnewisser, B.; Winnewisser, M. Investigation of the Ground Vibrational State of CD3OH by a New "Ritz" Program for Direct Energy Level Fitting. J. Mol. Spectrosc. 1994, 167, 156-175. doi:10.1006/jmsp.1994.1223.

115. Mukhopadhyay, I.; Duan, Y.; Takagi, K. High resolution spectroscopy of $\mathrm{CH}_{3} \mathrm{OD}$ involving the ground and first excited torsional states in the vibrational ground state. Spectrochim. Acta. A. Mol. Biomol. Spectrosc. 1998, 54, 1325-1335. doi:10.1016/S13861425(98)00041-9.

116. Xu, L.H.; Müller, H.; van der Tak, F.; Thorwirth, S. The millimeter-wave spectrum of perdeuterated methanol, CD3OD. J. Mol. Spectrosc. 2004, 228, 220-229. doi:10.1016/j.jms.2004.04.015.

117. Margulès, L.; McGuire, B.A.; Senent, M.L.; Motiyenko, R.A.; Remijan, A.; Guillemin, J.C. Submillimeter spectra of 2hydroxyacetonitrile (glycolonitrile; $\mathrm{HOCH}_{2} \mathrm{CN}$ ) and its searches in GBT PRIMOS observations of Sgr B2(N). Astron. Astrophys. 2017, 601, A50. doi:10.1051/0004-6361/201628551.

118. Cazzoli, G.; Lister, D.G.; Mirri, A.M. Rotational isomerism and barriers to internal rotation in hydroxyacetonitrile from microwave spectroscopy. J. Chem. Soc. Faraday Trans. 2 1973, 69, 569. doi:10.1039/f29736900569.

119. Motiyenko, R.A.; Margulès, L.; Senent, M.L.; Guillemin, J.C. Internal Rotation of OH Group in 4-Hydroxy-2-butynenitrile Studied by Millimeter-Wave Spectroscopy. J. Phys. Chem. A 2018, 122, 3163-3169. doi:10.1021/acs.jpca.7b12051.

120. Melandri, S.; Favero, P.G.; Caminati, W. Detection of the syn conformer of allyl alcohol by free jet microwave spectroscopy. Chem. Phys. Lett. 1994, 223, 541-545. doi:10.1016/0009-2614(94)00478-1.

121. Motiyenko, R.A.; Margulès, L.; Despois, D.; Guillemin, J.C. Laboratory spectroscopy of methoxymethanol in the millimeter-wave range. Phys. Chem. Chem. Phys. 2018, 20, 5509-5516. doi:10.1039/C7CP05932A.

122. Suenram, R.; Lovas, F.; Pickett, H. Fluoromethanol: Synthesis, microwave spectrum, and dipole moment. J. Mol. Spectrosc. 1986, 119, 446-455. doi:10.1016/0022-2852(86)90039-1.

123. Xu, L.H.; Fraser, G.T.; Lovas, F.J.; Suenram, R.D.; Gillies, C.W.; Warner, H.E.; Gillies, J.Z. The microwave spectrum and OH internal rotation dynamics of gauche -2,2,2-trifluoroethanol. J. Chem. Phys. 1995, 103, 9541-9548. doi:10.1063/1.469968.

124. Cohen, E.; Drouin, B.; Valenzuela, E.; Woods, R.; Caminati, W.; Maris, A.; Melandri, S. The rotational spectrum of tertiary-butyl alcohol. J. Mol. Spectrosc. 2010, 260, 77-83. doi:10.1016/j.jms.2009.11.010.

125. Li, W.; Spada, L.; Evangelisti, L.; Caminati, W. Conformational Equilibrium and Potential Energy Functions of the O-H Internal Rotation in the Axial and Equatorial Species of 1-Methylcyclohexanol. J. Phys. Chem. A 2016, 120, 4338-4342. doi:10.1021/acs.jpca.6b03854.

126. Leonov, A.; Marstokk, K.M.; de Meijere, A.; Møllendal, H. Microwave Spectrum, Conformational Equilibrium, Intramolecular Hydrogen Bonding, Tunneling, and Quantum Chemical Calculations for 1-Ethenylcyclopropan-1-ol. J. Phys. Chem. A 2000, 104, 4421-4428. doi:10.1021/jp9940755. 